Research Article

\title{
Transceiver Designs for MIMO Relaying Systems with Imperfect Channel State Information
}

\author{
Bridget Durowaa Antwi-Boasiako $\mathbb{D}^{1},{ }^{1}$ Changick Song $\mathbb{D},{ }^{2}$ and Kyoung-Jae Lee $\mathbb{D}^{1}$ \\ ${ }^{1}$ Department of Electronics and Control Engineering, Hanbat National University, 125 Dongseo-Daero, Yuseong-gu, \\ Daejeon, Republic of Korea \\ ${ }^{2}$ Department of Electronic Engineering, Korea National University of Transportation, \\ 50 Daehak-ro, Daesowon-myeon, Chungju, Chungcheongbuk-do, Republic of Korea \\ Correspondence should be addressed to Kyoung-Jae Lee; kyoungjae@hanbat.ac.kr
}

Received 16 October 2017; Revised 19 April 2018; Accepted 15 May 2018; Published 19 July 2018

Academic Editor: Jose F. Monserrat

Copyright (C) 2018 Bridget Durowaa Antwi-Boasiako et al. This is an open access article distributed under the Creative Commons Attribution License, which permits unrestricted use, distribution, and reproduction in any medium, provided the original work is properly cited.

\begin{abstract}
We focus on imperfect channel state information (ICSI) in closed-loop multiple-input multiple-output (MIMO) amplify-andforward (AF) relaying systems. First, near-optimal closed-form solutions for transceiver designs are provided for the source-torelay-to-destination link with ICSI at all nodes. Next, considering a nonnegligible direct link with ICSI available only at the relay and destination and no CSI or ICSI at the source, near-optimal designs are proposed. The Wiener or minimum mean square error (MMSE) filter is employed at the destination to estimate the transmitted signal. The effect of CSI mismatch on the performance of the proposed scheme is then shown through simulations.
\end{abstract}

\section{Introduction}

The design of future wireless networks will require that systems provide high throughput, reliable transmission, and broader coverage. According to research, these benefits are realized when multiple-input multiple-output (MIMO) systems are utilized with relays $[1,2]$. Different relay protocols are available and can be classified based on their operation. One commonly known relay protocol is the regenerative or decode-and-forward (DF) relaying scheme, which allows the relay to decode or regenerate the original information from the previous node before retransmitting it to the subsequent node. Another protocol, known as nonregenerative or amplify-and-forward (AF) relaying scheme, however, does not allow decoding of the received signal but only amplifies and retransmits it. Although there are other protocols, AF relay nodes are normally employed for reduced complexity and easy implementation. Relays are also classified as either full-duplex relays or half-duplex relays. Full-duplex relays transmit and receive data simultaneously while half-duplex systems do not $[3,4]$.
The benefits of MIMO systems are enhanced not only when information about the channel, that is, channel state information (CSI), is known at the receiver but also at the transmitter [5]. When there is channel state information at the transmitter (CSIT), a processing technique, known as precoding, exploits the information about the channel by operating on the signal before transmission to better suit it for channel conditions. The transmitter cannot directly access its own CSI, thus CSIT can only be acquired indirectly. In practice, the quality of CSI obtained is often imperfect and degraded due to quantization errors, feedback delay, and channel estimation errors [6, 7]. The imperfect CSI (ICSI) causes performance degradation and since these errors are inevitable, it is necessary to formulate robust designs to combat their effects [3].

There have been many works on AF MIMO relaying systems. In [8, 9], precoder designs for a three-node MIMO AF system are studied. Also, there have been works based on the two main kinds of criteria for transceiver designs, which are capacity maximization and mean square error minimization. In [10-12], optimizing the capacity between the 
source and the destination is considered. In addition, asymptotic ergodic capacity is analyzed with a linear processing at the relay in [13]. Optimization designs based on MSE criteria are formulated for the two-way AF relaying channel in [14], and Guan and Luo [15] address the problem of transceiver designs by jointly minimizing the MSE of symbol estimations. These existing schemes, however, do not consider the scenario where there is ICSI. In the presence of ICSI, robust designs have been studied in [16], but this study does not consider the MSE decomposition and relaxation property [3].

One of the most widely considered power constraints in transmission is norm constraints (NC), which limit the expected norm of the transmit vector. In practice, however, the NC may not be suitable since each antenna element is normally equipped with its own power amplifier, and thus its power is limited individually. To better suit practical systems, the design is also subjected to maximum eigenvalue constraints (MVC) ensuring peak power constraint, which limits the maximum of the output power and thus satisfies the power limits on each antenna [17].

In this paper, we provide detailed closed-form expressions for robust joint MMSE designs of source-relay and relay-destination links for AF relaying system considering $\mathrm{NC}$ and MVC and account for the practical scenario of a nonnegligible direct link, which has not been considered in [3]. We first allow CSI at all nodes and secondly, only at relay-destination nodes. The MMSE receiver is employed due to its simplicity and effectiveness [18]. It is also known that MMSE mitigates both interference and noise compared to other linear detectors such as zero forcing (ZF), which cancels interference but enhances noise power [19]. The analysis in this paper is facilitated by employing MMSE decomposition property found in $[3,20]$. This property makes use of the (relatively) high SNR assumption stated in [21] to decouple the transmitter and relay optimization problems. Based on extensive simulations (see Figures 2 and 3 of [2] and Figure 3 of [3]), although the proposed scheme employs high SNR approximation in its derivation, it exhibits very little loss in performance when compared with the optimal design over all SNR range thus confirming the optimality of the MSE decomposition method and providing lower complexity [3]. We also use the bit error rate (BER) criteria to minimize the BER for a certain MSE. The organization of this paper is as follows: Section 2 describes the system model. In Section 3, assuming negligible direct link, the optimal receive filter and transceiver with imperfect CSI are derived and the proposed designs for source-relaydestination joint and relay-destination joint MMSE designs are presented. Section 4 continues with the discussion of the model including the direct path between the source and destination with no precoder at the input and presents the proposed design. Simulation results are shown in Section 5, and conclusions are drawn in Section 6.

Throughout this paper, bold letters represent vectors, normal letters indicate scalar quantities, and bold uppercase letters designate matrices. The superscripts $(\cdot)^{\dagger},(\cdot)^{T}$, and $(\cdot)^{*}$ stand for conjugate transpose, transpose, and elementwise conjugate, respectively; $\varepsilon(\cdot)$ represents the expectation

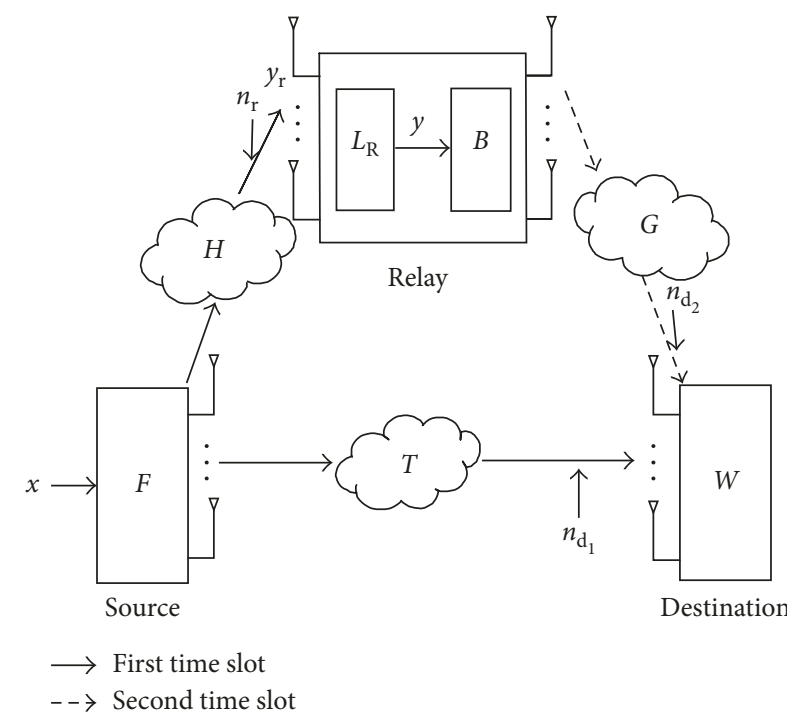

Figure 1: System description for the nonregenerative MIMO relaying system.

operator; $\mathbf{I}_{N}$ is an $N \times N$ identity matrix; and $\operatorname{tr}(\mathbf{A})$ indicates the trace of a matrix $\mathbf{A}$. The minimum and maximum singular value of a matrix $\mathbf{A}$ are denoted as $\lambda_{\text {min }}(\mathbf{A})$ and $\lambda_{\max }(\mathrm{A})$. The matrix constructed by the first $N_{\mathrm{s}}$ columns of a matrix $\mathbf{A}$ is denoted as $\overline{\mathbf{A}}$.

\section{System Model for MIMO AF Relaying}

In this section, we present the general system model for a nonregenerative MIMO relaying system with precoded input. A half-duplex AF relay is used for data transmission which occurs in two separate time slots as seen in Figure 1. It is assumed that the source, relay, and destination node use $N_{\mathrm{t}}, N_{\mathrm{r}}$, and $N_{\mathrm{d}}$ antennas, respectively. The MIMO system allows the use of spatial multiplexing (SM) which transmits $N_{s}$ data streams simultaneously, with the assumption that $N_{\mathrm{s}} \leq \min \left\{N_{\mathrm{t}}, N_{\mathrm{r}}, N_{\mathrm{d}}\right\}$.

During the first time slot, a precoder $\mathbf{F} \in \mathbb{C}^{N_{\mathrm{t}} \times N_{\mathrm{s}}}$ multiplies the source data vector $\mathbf{x}$ and is transmitted to the relay node and also to the destination. The source power is given by $\varepsilon\left\{\mathbf{x x}^{\dagger}\right\}=\sigma_{x}^{2} \mathbf{I}_{N_{s}}$. The received signal vectors at the relay and destination nodes, $\mathbf{y}_{\mathrm{r}} \in \mathbb{C}^{N_{\mathrm{r}} \times 1}$ and $\mathbf{y}_{\mathrm{d}_{1}} \in \mathbb{C}^{N_{\mathrm{d}} \times 1}$, are given, respectively, as

$$
\begin{aligned}
\mathbf{y}_{\mathrm{r}} & =\mathbf{H F x}+\mathbf{n}_{\mathrm{r}}, \\
\mathbf{y}_{\mathrm{d}_{1}} & =\mathbf{T F x}+\mathbf{n}_{\mathrm{d}_{1}},
\end{aligned}
$$

where $\mathbf{H} \in \mathbb{C}^{N_{\mathrm{r}} \times N_{\mathrm{t}}}$ is the first hop channel matrix from the source to the relay and $\mathbf{T} \in \mathbb{C}^{N_{\mathrm{d}} \times N_{\mathrm{t}}}$ is the source-to-destination channel matrix. The noise vectors at the relay and destination are indicated as $\mathbf{n}_{\mathrm{r}} \in \mathbb{C}^{N_{\mathrm{r}} \times 1}$ with $\varepsilon\left\{\mathbf{n}_{\mathrm{r}} \mathbf{n}_{\mathrm{r}}^{\dagger}\right\}=\sigma_{n_{\mathrm{r}}}^{2} \mathbf{I}_{N_{\mathrm{r}}}$ and $\mathbf{n}_{\mathrm{d}_{1}} \in \mathbb{C}^{N_{\mathrm{d}} \times 1}$ with $\varepsilon\left\{\mathbf{n}_{\mathrm{d}_{1}} \mathbf{n}_{\mathrm{d}_{1}}^{\dagger}\right\}=\sigma_{n_{\mathrm{d}_{1}}}^{2} \mathbf{I}_{N_{\mathrm{d}}}$, respectively. In the second time slot, the signal is amplified by the relay transceiver, $\mathbf{Q} \in \mathbb{C}^{N_{\mathrm{r}} \times N_{\mathrm{r}}}$, and transmitted to the destination. The transceiver has the form $\mathbf{Q}=\mathbf{B L}_{\mathrm{R}}$, where $\mathbf{B} \in \mathbb{C}^{N_{\mathrm{r}} \times N_{\mathrm{s}}}$ is the relay precoder and $\mathbf{L}_{\mathrm{R}} \in \mathbb{C}^{N_{s} \times N_{\mathrm{r}}}$ is the relay receiver, without loss of generality. 
The received signal at the destination is then given by

$$
\begin{aligned}
\mathbf{y}_{\mathrm{d}_{2}} & =\mathbf{G Q H F x}+\mathbf{G Q} \mathbf{n}_{\mathrm{r}}+\mathbf{n}_{\mathrm{d}_{2}}, \\
& =\mathbf{G Q H F x}+\mathbf{n},
\end{aligned}
$$

where $\mathbf{G} \in \mathbb{C}^{N_{\mathrm{d}} \times N_{\mathrm{r}}}$ is the second hop channel matrix and $\mathbf{n}=$ $\mathbf{G Q n} \mathbf{n}_{\mathrm{r}}+\mathbf{n}_{\mathrm{d}_{2}}$ is defined as the effective noise vector.

To introduce the imperfection caused by channel estimation errors, we adopt error model used in [22, 23]. The channel model is thus modified as

$$
\begin{aligned}
& \mathbf{H}=\widehat{\mathbf{H}}+\mathbf{E}_{\mathrm{H}}, \\
& \mathbf{T}=\widehat{\mathbf{T}}+\mathbf{E}_{\mathrm{T}}, \\
& \mathbf{G}=\widehat{\mathbf{G}}+\mathbf{E}_{\mathrm{G}},
\end{aligned}
$$

where $\widehat{\mathbf{H}}, \widehat{\mathbf{T}}$, and $\widehat{\mathbf{G}}$ are the estimated channels while $\mathbf{E}_{\mathrm{H}}, \mathbf{E}_{\mathrm{T}}$, and $\mathbf{E}_{\mathrm{G}}$ are the corresponding channel estimation errors with elements which are zero mean Gaussian random variables with variances given as $\sigma_{\mathrm{E}_{\mathrm{H}}}^{2}, \sigma_{\mathrm{E}_{\mathrm{T}}}^{2}$, and $\sigma_{\mathrm{E}_{\mathrm{G}}}^{2}$, respectively. The variances reflect the quality of the channel estimation $[22,24]$.

The two signals received at the destination over the two consecutive time slots can be combined into signal vector $\mathbf{y}_{\mathrm{d}} \in \mathbb{C}^{2 N_{\mathrm{d}} \times 1}$. This is given as

$$
\mathbf{y}_{\mathrm{d}}=\left[\begin{array}{l}
\mathbf{y}_{\mathrm{d}_{1}} \\
\mathbf{y}_{\mathrm{d}_{2}}
\end{array}\right]=\left[\begin{array}{c}
\mathbf{T F} \\
\mathbf{G Q H F}
\end{array}\right] \mathbf{x}+\left[\begin{array}{c}
\mathbf{n}_{\mathrm{d}_{1}} \\
\mathbf{n}
\end{array}\right] .
$$

The transmitted signal estimate obtained after employing a Wiener filter $\mathbf{W} \in \mathbb{C}^{N_{\mathrm{s}} \times 2 N_{\mathrm{d}}}$ is given as $\widetilde{\mathbf{y}}_{\mathrm{D}}=\mathbf{W} \mathbf{y}_{\mathrm{d}}=$ $\mathbf{W}_{1} \mathbf{y}_{\mathrm{d}_{1}}+\mathbf{W}_{2} \mathbf{y}_{\mathrm{d}_{2}}$, where $\widetilde{\mathbf{y}}_{\mathrm{d}} \in \mathbb{C}^{N_{\mathrm{s}} \times 1}$ and $\mathbf{W}_{1}$ and $\mathbf{W}_{2} \in \mathbb{C}^{N_{\mathrm{s}} \times N_{\mathrm{d}}}$ are submatrices of $\mathbf{W}$ for the direct and relay links, respectively. This model can be reduced to the case where there is no direct link by removing the source-todestination link.

\section{MIMO AF Relay without Direct Link}

The discussion in the following section assumes a negligible direct link. Thus, there is no direct transmission from the source to the destination. The total transmit power at the source is defined as $P_{\mathrm{T}} \triangleq \varepsilon\|\mathbf{F} \mathbf{x}\|^{2}=\sigma_{x}^{2} N_{s}$, assuming $\operatorname{tr}\left(\mathbf{F F}^{\dagger}\right)=$ $N_{s}$. The signal received at the destination is denoted as $\mathbf{y}_{\mathrm{D}}$ to distinguish that in the direct link case. Also, the final observation $\widetilde{\mathbf{y}}_{\mathrm{D}}$ is given as $\widetilde{\mathbf{y}}_{\mathrm{D}}=\mathbf{W}_{\mathrm{D}} \mathbf{y}_{\mathrm{D}}$, where $\mathbf{W}_{\mathrm{D}}$ is the linear Wiener filter.

The singular value decomposition (SVD) is used to convert the MIMO channel into multiple parallel independent subchannels $[25,26]$. The SVD of the channels $\mathbf{H}$ and $\mathbf{G}$ are given as $\mathbf{H}=\mathbf{U}_{\mathrm{h}} \boldsymbol{\Phi} \mathbf{V}_{\mathrm{h}}^{\dagger}$ and $\mathbf{G}=\mathbf{U}_{\mathrm{g}} \boldsymbol{\Omega} \mathbf{V}_{\mathrm{g}}^{\dagger}$, respectively, and $\mathbf{U}_{\mathrm{h}}, \mathbf{V}_{\mathrm{h}}, \mathbf{U}_{\mathrm{g}}$, and $\mathbf{V}_{\mathrm{g}}$ are unitary matrices. The real and nonnegative diagonal matrices, $\Phi \in \mathbb{R}^{N_{\mathrm{r}} \times N_{\mathrm{t}}}$ and $\Omega \in \mathbb{R}^{N_{\mathrm{d}} \times N_{\mathrm{r}}}$, have respective singular values which are given by $\phi_{i}$ for $i=1,2, \ldots, \min \left(N_{\mathrm{t}}, N_{\mathrm{r}}\right)$ and $\omega_{i}$ for $i=1,2, \ldots, \min \left(N_{\mathrm{r}}, N_{\mathrm{d}}\right)$ and are arranged in a descending order on the main diagonal. The $N_{s}$ dimensional square diagonal matrices $\overline{\boldsymbol{\Phi}}$ and $\overline{\boldsymbol{\Omega}}$ are also defined as $\overline{\boldsymbol{\Phi}} \triangleq \operatorname{diag}$ $\left\{\phi_{1}, \phi_{2}, \ldots, \phi_{N_{s}}\right\}$ and $\bar{\Omega} \triangleq \operatorname{diag}\left\{\omega_{1}, \omega_{2}, \ldots, \omega_{N_{s}}\right\}$, respectively.

\subsection{Problem Formulation}

3.1.1. Optimum Destination Receiver. Armed with global ICSI, a Wiener filter is employed at the destination to minimize the expected mean squared error between the transmitted and detected symbols. The MMSE optimal receive filter (destination Wiener filter) is formulated as

$$
\widehat{\mathbf{W}}_{\mathrm{D}}=\arg \min _{\mathbf{W}_{\mathrm{D}}} \varepsilon\|\mathbf{e}\|^{2},
$$

where $\mathbf{e}$ is the error vector defined as $\mathbf{e} \triangleq \gamma^{-1} \widetilde{\mathbf{y}}_{\mathrm{D}}-\mathbf{x}=$ $\mathbf{W}_{\mathrm{D}} \dot{\mathbf{y}}_{\mathrm{D}}-\mathbf{x}$, with $\dot{\mathbf{y}}_{\mathrm{D}}=\gamma^{-1} \mathbf{y}_{\mathrm{D}}$. Here, $\gamma^{-1}$ is a scaling factor to simplify derivation.

Theorem 1. We first obtain the optimal receive filter $\widehat{\mathbf{W}}_{\mathrm{D}}$. Given specific $\mathbf{Q}, \mathbf{F}$, and $\gamma$, the filter is easily obtained as

$$
\widehat{\mathbf{W}}_{\mathrm{D}}=\gamma \sigma_{x}^{2}\left(\mathbf{F}^{\dagger} \widehat{\mathbf{H}}^{\dagger} \mathbf{Q}^{\dagger} \widehat{\mathbf{G}}^{\dagger}\right)\left[\left(\widehat{G} \mathbf{Q} \mathscr{A} \mathbf{Q}^{\dagger} \widehat{\mathbf{G}}^{\dagger}+\beta \mathbf{I}_{N_{\mathrm{d}}}\right)\right]^{-1},
$$

where we define $\beta=\left[\sigma_{n_{\mathrm{d}_{2}}}^{2}+\sigma_{\mathrm{E}_{\mathrm{T}}}^{2} \operatorname{tr}\left(\gamma^{2} \mathbf{B R}_{y} \mathbf{B}^{\dagger}\right)\right] . \mathbf{R}_{y}$ is the covariance matrix of the signal $\mathbf{y}$, where $\mathbf{R}_{y}=\varepsilon\left(\mathbf{y y}^{\dagger}\right)$. Here, $\mathbf{y}$ indicates the product of the relay receive filter $\mathrm{L}_{\mathrm{R}}$ and the signal received at the relay $\mathrm{y}_{\mathrm{r}}$ and also $\mathscr{A}$ is defined as $\mathscr{A}=\left[\sigma_{n_{\mathrm{r}}}^{2}+\sigma_{\mathrm{E}_{\mathrm{H}}}^{2} \operatorname{tr}\left(\sigma_{x}^{2} \mathbf{F F}^{\dagger}\right) \mathbf{I}_{N_{\mathrm{r}}}+\sigma_{x}^{2} \widehat{\mathbf{H}} \mathbf{F} \mathbf{F}^{\dagger} \widehat{\mathbf{H}}^{\dagger}\right]$.

Proof. See Appendix A.

3.1.2. Error Covariance Matrix $\mathbf{R}_{E}$. The error covariance matrix, also known as the MSE matrix, defined as $\mathbf{R}_{\mathrm{E}} \triangleq \varepsilon\left[\mathbf{e e}^{\dagger}\right]$ is given by

$$
\begin{aligned}
\mathbf{R}_{\mathrm{E}} & =\varepsilon\left[\left(\widehat{\mathbf{W}}_{\mathrm{D}} \dot{\mathbf{y}}_{\mathrm{D}}-\mathbf{x}\right)\left(\widehat{\mathbf{W}}_{\mathrm{D}} \dot{\mathbf{Y}}_{\mathrm{D}}-\mathbf{x}\right)^{\dagger}\right], \\
& =\left[\mathscr{H} \mathbf{R}^{-1} \mathscr{H}^{\dagger}+\left(1 / \sigma_{x}^{2}\right) \mathbf{I}_{N_{\mathrm{s}}}\right]^{-1},
\end{aligned}
$$

where $\mathscr{H}=\widehat{\mathbf{G}} \mathbf{Q} \widehat{\mathbf{H}} \mathbf{F}$ and $\mathbf{R}=\widehat{\mathbf{G}} \mathbf{Q}\left[\sigma_{n_{\mathrm{r}}}^{2}+\sigma_{\mathrm{E}_{\mathrm{H}}}^{2} \operatorname{tr}\left(\sigma_{x}^{2} \mathbf{F} \mathbf{F}^{\dagger}\right)\right] \mathbf{Q}^{\dagger} \widehat{\mathbf{G}}^{\dagger}+$ $\beta \mathbf{I}_{N_{\mathrm{d}}}$. Here, $\mathbf{R}$ is the equivalent noise covariance and the corresponding MSE is obtained by $\varepsilon\|\mathbf{e}\|^{2}=\operatorname{tr}\left(\mathbf{R}_{\mathrm{E}}\right)$.

3.1.3. Optimum Relay Transceiver. Subject to the relay power budget $P_{\mathrm{R}}$, the design of the optimal relay transceiver can be expressed as the following optimization problem:

$$
\begin{array}{ll}
\min _{\mathbf{Q}} & \varepsilon\|\mathbf{e}\|^{2}, \\
\text { s.t. } & \operatorname{tr}\left(\mathbf{Q} \mathscr{A} \mathbf{Q}^{\dagger}\right)=P_{\mathrm{R}} .
\end{array}
$$

The optimal relay transceiver can be found by formulating the cost function $\mathscr{C}$ with the Lagrangian multiplier $\lambda$ as

$$
\mathscr{C}=\varepsilon\|\mathbf{e}\|^{2}+\lambda\left[\operatorname{tr}\left(\mathbf{Q} \mathscr{A} \mathbf{Q}^{\dagger}\right)-P_{\mathrm{R}}\right]
$$

Theorem 2. The following theorem is formulated to get the optimal relay structure from (9). For a given source precoder $\mathbf{F}$ and filter $\mathbf{W}_{\mathrm{D}}$, the optimal relay transceiver has the form $\widehat{\mathbf{Q}}=\gamma \widehat{\mathbf{Q}}=\gamma \mathbf{B} \mathbf{L}_{\mathrm{R}}$, where $\gamma, \mathbf{B}$, and $\mathbf{L}_{\mathrm{R}}$ are calculated as 


$$
\begin{aligned}
\gamma= & \sqrt{\frac{P_{\mathrm{R}}}{\operatorname{tr}\left(\widetilde{\mathbf{Q}} \mathscr{A} \widetilde{\mathbf{Q}}^{\dagger}\right)}}, \\
\mathbf{B}= & \widehat{\mathbf{G}}^{\dagger} \mathbf{W}_{\mathrm{D}}^{\dagger}\left[\mathbf{W}_{\mathrm{D}} \widehat{\mathbf{G}} \widehat{\mathbf{G}}^{\dagger} \mathbf{W}_{\mathrm{D}}^{\dagger}\right. \\
& \left.+\left(\frac{\operatorname{tr}\left(\sigma_{n_{2}}^{2} \mathbf{W}_{\mathrm{D}} \mathbf{W}_{\mathrm{D}}^{\dagger}\right)}{P_{\mathrm{R}}}+\operatorname{tr}\left(\sigma_{\mathrm{E}_{\mathrm{T}}^{2}}^{2} \mathbf{W}_{\mathrm{D}} \mathbf{W}_{\mathrm{D}}^{\dagger}\right)\right) \mathbf{I}_{N_{\mathrm{s}}}\right]^{-1}, \\
\mathbf{L}_{\mathrm{R}}= & {\left[\mathbf{F}^{\dagger} \hat{\mathbf{H}}^{\dagger} \widehat{\mathbf{H}} \mathbf{F}+\left(\frac{\sigma_{n_{\mathrm{r}}}^{2}}{\sigma_{x}^{2}}\right) \mathbf{I}_{N_{\mathrm{s}}}+\left(\frac{\sigma_{\mathrm{E}_{\mathrm{H}}}^{2}}{\sigma_{x}^{2}}\right) \operatorname{tr}\left(\sigma_{x}^{2} \mathbf{F} \mathbf{F}^{\dagger}\right) \mathbf{I}_{N_{\mathrm{s}}}\right]^{-1} \mathbf{F}^{\dagger} \widehat{\mathbf{H}}^{\dagger} . }
\end{aligned}
$$

Proof. See Appendix B.

Here, it should be noted that the relay power normalizing coefficient is given by $\gamma, \mathbf{B}$ is the relay transmit Wiener filter for the second hop channel $\mathbf{G}$, and $\mathrm{L}_{\mathrm{R}}$ is the relay receive Wiener filter (R-WF) for the first hop channel.

\subsubsection{Decomposition of $\mathbf{R}_{\mathrm{E}}$}

Theorem 3. We now obtain the following form of error covariance matrix $\mathbf{R}_{\mathrm{E}}$ using the MSE decomposition property. This allows the error matrix $\mathbf{R}_{\mathrm{E}}$ to be expressed as a sum of two separate error matrices given the optimal relay transceiver $\hat{\mathbf{Q}}$ and destination filter $\widehat{\mathbf{W}}_{\mathrm{D}}$ as follows:

$$
\begin{aligned}
\mathbf{R}_{\mathrm{E}}(\mathbf{F}, \mathbf{B})= & \left(\sigma_{n_{\mathrm{r}}}^{2}+\sigma_{\mathrm{E}_{\mathrm{H}}}^{2} P_{\mathrm{T}}\right)\left(\mathbf{F}^{\dagger} \hat{\mathbf{H}}^{\dagger} \hat{\mathbf{H}} \mathbf{F}+\frac{\sigma_{n_{\mathrm{r}}}^{2}+\sigma_{\mathrm{E}_{\mathrm{H}}}^{2} P_{\mathrm{T}}}{\sigma_{x}^{2}} \mathbf{I}_{N_{\mathrm{s}}}\right)^{-1} \\
& +\beta\left(\gamma^{2} \mathbf{B}^{\dagger} \hat{\mathbf{G}}^{\dagger} \hat{\mathbf{G}} \mathbf{B}+\beta \mathbf{R}_{y}^{-1}\right)^{-1}
\end{aligned}
$$

where $\mathbf{R}_{y}$ is the relay covariance matrix of signal $\mathbf{y}$.

Proof. See Appendix C.

It can be observed from the above that only precoder $\mathbf{F}$ can be found in the first MSE decomposition term. Although the second term is also related to the precoder $\mathbf{F}$, this can be relaxed to allow the two precoders to be optimized independently of each other. This will be discussed briefly in the next subsection below.

3.2. Source-Relay-Destination Joint MMSE Design. The joint transceiver design seeks to minimize the MSE by the design of the precoding matrix $\mathbf{F}$ and the amplifying relay matrix $\mathbf{Q}$ under total power constraints at the source and relay nodes taking into account the CSI mismatch.

3.2.1. Norm Power Constraint (Robust SRD-NC/BNC). The joint design considering norm power constraints when ICSI is known at all nodes is referred to as robust joint source-relay-destination MMSE designs with NC (Robust SRD-NC). Using the NC, the problem for minimizing the MSE can be formulated as

$$
\begin{array}{ll}
\underset{(\mathbf{F}, \mathbf{B})}{\min } & \mathbf{R}_{\mathrm{E}}(\mathbf{F}, \mathbf{B}), \\
\text { s.t. } & \operatorname{tr}\left(\sigma_{x}^{2} \mathbf{F} \mathbf{F}^{\dagger}\right)=P_{\mathrm{T}}, \\
\text { s.t. } & \operatorname{tr}\left(\mathbf{B R}_{y} \mathbf{B}^{\dagger}\right)=P_{\mathrm{R}} .
\end{array}
$$

The constraints above are set to full power since this is optimal in terms of MSE [2, 27].

As mentioned in the previous section, an approximate approach is employed to 'relax' or 'eliminate' the mutual exclusivity of the matrices $\mathbf{F}$ and $\mathbf{B}$ in order to obtain a closed-form solution. From (C.2), it can be easily checked that under the assumption that $\sigma_{x}^{2} /\left[\sigma_{n_{\mathrm{r}}}^{2}+\sigma_{\mathrm{E}_{\mathrm{H}}}^{2} \operatorname{tr}\left(\sigma_{x}^{2} \mathbf{F F}^{\dagger}\right)\right] \gg 1$, $\mathbf{R}_{y}$ rapidly approaches the identity matrix $\sigma_{x}^{2} \mathbf{I}_{N_{s}}$. This allows the source precoding matrix $\mathbf{F}$ to be determined independently of $\mathbf{B}$.

Thus, the problem for minimizing MMSE with norm power constraint in (12) can be separated as

$$
\begin{array}{ll}
\min _{\mathbf{F}} & \operatorname{tr}\left[\left(\sigma_{n_{\mathrm{r}}}^{2}+\sigma_{\mathrm{E}_{\mathrm{H}}}^{2} P_{\mathrm{T}}\right)\left(\mathbf{F}^{\dagger} \hat{\mathbf{H}}^{\dagger} \hat{\mathbf{H}} \mathbf{F}+\frac{\left(\sigma_{n_{\mathrm{r}}}^{2}+\sigma_{\mathrm{E}_{\mathrm{H}}}^{2} P_{\mathrm{T}}\right)}{\sigma_{x}^{2}} \mathbf{I}_{N_{\mathrm{s}}}\right)^{-1}\right], \\
\text { s.t. } & \operatorname{tr}\left(\sigma_{x}^{2} \mathbf{F} \mathbf{F}^{\dagger}\right)=P_{\mathrm{T}}, \\
\min _{\mathbf{B}} \operatorname{tr}\left[\left(\sigma_{n_{\mathrm{dx}_{2}}}^{2}+\sigma_{\mathrm{E}_{\mathrm{T}}}^{2} P_{\mathrm{R}}\right)\left(\mathbf{B}^{\dagger} \widehat{\mathbf{G}}^{\dagger} \hat{\mathbf{G}} \mathbf{B}+\left(\sigma_{n_{\mathrm{d}_{2}}}^{2}+\sigma_{\mathrm{E}_{\mathrm{T}}}^{2} P_{\mathrm{R}}\right) \mathbf{R}_{y}^{-1}\right)^{-1}\right],
\end{array}
$$$$
\text { s.t. } \quad \operatorname{tr}\left(\mathbf{B R}_{y} \mathbf{B}^{\dagger}\right)=P_{\mathbf{R}} \text {. }
$$

Using [28], which shows that a diagonalized channel structure optimizes a trace function, the optimal solutions of the above equations are assumed to be of the form $\widehat{\mathbf{F}}=\overline{\mathbf{V}}_{\mathrm{h}} \boldsymbol{\Delta}_{f}$ and $\widehat{\mathbf{B}}=\overline{\mathbf{V}}_{\mathrm{g}} \boldsymbol{\Delta}_{b}$. Here, $\boldsymbol{\Delta}_{f}$ and $\boldsymbol{\Delta}_{b}$ represent the power loading matrices and are defined as $\Delta_{f} \triangleq \operatorname{diag}\left\{f_{1}, f_{2}, \ldots, f_{N_{\mathrm{s}}}\right\}$ and $\Delta_{b} \triangleq \operatorname{diag}\left\{b_{1}, b_{2}, \ldots, b_{N_{\mathrm{s}}}\right\}$. The power loading is done with the following water-pouring like solutions:

$$
\begin{aligned}
& \left|f_{i}\right|^{2}=\frac{1}{\sigma_{x}^{2} \phi^{2}}\left(\sqrt{\frac{\sigma_{x}^{2} \phi^{2}\left(\sigma_{n_{\mathrm{r}}}^{2}+\sigma_{\mathrm{E}_{\mathrm{H}}}^{2} P_{\mathrm{T}}\right)}{v}}-\left(\sigma_{n_{\mathrm{r}}}^{2}+\sigma_{\mathrm{E}_{\mathrm{H}}}^{2} P_{\mathrm{T}}\right)\right)^{+}, \\
& \left|b_{i}\right|^{2}=\frac{1}{\omega^{2} r_{i}}\left(\sqrt{\frac{r_{i} \omega_{i}^{2}\left(\sigma_{n_{\mathrm{d}_{2}}^{2}}^{2}+\sigma_{\mathrm{E}_{\mathrm{T}}}^{2} P_{\mathrm{R}}\right)}{\tau}}-\left(\sigma_{n_{\mathrm{d}_{2}}^{2}}^{2}+\sigma_{\mathrm{E}_{\mathrm{T}}}^{2} P_{\mathrm{R}}\right)\right)^{+},
\end{aligned}
$$

where $(x)^{+} \triangleq \max (x, 0), v$ and $\tau$ are chosen to meet the power constraint (12), and $r_{i}$ stands for the $i$ th diagonal term of $\mathbf{R}_{y}$.

We briefly introduce another design criterion known as the BER-based criterion to generate the precoding matrices. This is obtained as a consequence of minimizing the maximum MSE problem in the paragraph above. Studies in $[14,28]$ show that a discrete Fourier transform (DFT) or Hadamard matrix is applied on the diagonalized error 
matrix to minimize BER by making all diagonal elements of the MSE matrix have the same value while maintaining the MSE. The precoders are therefore assumed as $\widehat{\mathbf{F}}=\overline{\mathbf{V}}_{\mathrm{h}} \boldsymbol{\Delta}_{f} \mathbf{Z}$ and $\widehat{\mathbf{B}}=\overline{\mathbf{V}}_{\mathrm{g}} \boldsymbol{\Delta}_{b} \mathbf{Z}$, where $\mathbf{Z}$ is the $N_{\mathrm{s}} \times N_{\mathrm{s}}$ DFT matrix. This BER minimization is called robust SRD-BNC.

3.3.2. Maximum Eigenvalue Constraint (Robust SRD-MV). This optimization problem for the robust source-relaydestination designs with the MVC (robust SRD-MV) designed to limit the peak power of the output can be defined as follows:

$\min _{\mathbf{F}} \operatorname{tr}\left[\left(\sigma_{n_{\mathrm{r}}}^{2}+\sigma_{\mathrm{E}_{\mathrm{H}}}^{2} P_{\mathrm{T}}\right)\left(\mathbf{F}^{\dagger} \widehat{\mathbf{H}}^{\dagger} \widehat{\mathbf{H}} \mathbf{F}+\frac{\left(\sigma_{n_{\mathrm{r}}}^{2}+\sigma_{\mathrm{E}_{\mathrm{H}}}^{2} P_{\mathrm{T}}\right)}{\sigma_{x}^{2}} \mathbf{I}_{N_{\mathrm{s}}}\right)^{-1}\right]$,

s.t. $\quad \lambda_{\max }\left(\sigma_{x}^{2} \mathbf{F F}^{\dagger}\right)=L_{\mathrm{T}}$,

$\min _{\mathbf{B}} \operatorname{tr}\left[\left(\sigma_{n_{\mathrm{d}_{2}}}^{2}+\sigma_{\mathrm{E}_{\mathrm{T}}}^{2} P_{\mathrm{R}}\right)\left(\mathbf{B}^{\dagger} \widehat{\mathbf{G}}^{\dagger} \widehat{\mathbf{G}} \mathbf{B}+\left(\sigma_{n_{\mathrm{d}_{2}}}^{2}+\sigma_{\mathrm{E}_{\mathrm{T}}}^{2} P_{\mathrm{R}}\right) \mathbf{R}_{y}^{-1}\right)^{-1}\right]$,

s.t. $\quad \lambda_{\max }\left(\mathbf{B R}_{y} \mathbf{B}^{\dagger}\right)=\max _{i}\left(r_{i}\left|b_{i}\right|^{2}\right)=L_{\mathrm{R}}$.

The solution for the source optimal precoder is given as $\hat{\mathbf{F}}=\sqrt{L_{\mathrm{T}} / \sigma_{x}^{2}} \overline{\mathbf{V}}_{\mathrm{h}}$ and that of the optimal relay transmit filter is given as $\widehat{\mathbf{B}}=\overline{\mathbf{V}}_{\mathrm{h}} \boldsymbol{\Delta}_{b}$, where the ith entry of $\boldsymbol{\Delta}_{b}$ is given as $b_{i}=\sqrt{L_{\mathrm{R}} / r_{i}}$.

\subsection{Relay-Destination Joint MMSE Design}

3.3.1. Norm Power Constraint (Robust RD-NC). In this scheme, there is no available CSI at the source (open-loop SM case). Thus, the source precoder $\mathbf{F}$ is set as $\mathbf{F}=\mathbf{I}_{N_{\mathrm{t}}}$. Global ICSI, however, is only available at the relay and the destination to compute the relay matrix and the receive Weiner filter. The optimization problem in (12) is thus written as

$$
\begin{array}{ll}
\min _{\mathbf{B}} & \operatorname{tr}\left(\mathrm{MSE}_{\mathscr{G}}\right), \\
\text { s.t. } & \operatorname{tr}\left(\mathbf{B R}_{y} \mathbf{B}^{\dagger}\right)=P_{\mathrm{R}},
\end{array}
$$

where we define MSE as $\operatorname{MSE}_{\mathscr{G}} \triangleq\left(\sigma_{n_{\mathrm{d}}}^{2}+\sigma_{\mathrm{E}_{\mathrm{T}}}^{2} P_{\mathrm{R}}\right)$ $\left(\mathbf{B}^{\dagger} \widehat{\mathbf{G}}^{\dagger} \widehat{\mathbf{G B}}+\left(\sigma_{n_{\mathrm{d}_{2}}}^{2}+\sigma_{\mathrm{E}_{\mathrm{T}}}^{2} P_{\mathrm{R}}\right) \mathbf{R}_{y}^{-1}\right)^{-1}$. The SVD of $\mathbf{R}_{y}=\mathbf{V}_{\mathrm{h}} \Sigma \mathbf{V}_{\mathrm{h}}^{\dagger}$ and we assume the optimal $\widehat{\mathbf{B}}=\overline{\mathbf{V}}_{\mathrm{g}} \boldsymbol{\Xi} \mathbf{V}_{\mathrm{h}}^{\dagger}$, where $\boldsymbol{\Sigma}=$ $\sigma_{x}^{4} \boldsymbol{\Phi}^{2}\left(\sigma_{x}^{2} \boldsymbol{\Phi}^{2}+\sigma_{n_{\mathrm{r}}}^{2} \mathbf{I}_{N_{\mathrm{s}}}+\sigma_{E_{\mathrm{H}}}^{2} \operatorname{tr}\left(\sigma_{x_{1}}^{2} \mathbf{I}_{N_{\mathrm{s}}}\right) \mathbf{I}_{N_{\mathrm{s}}}\right)^{-1}, \widetilde{\Sigma}^{\mathrm{h}}=\sigma_{x_{1}}^{2} \Sigma$, and $\Xi$ is an $N_{\mathrm{s}} \times N_{\mathrm{s}}$ diagonal matrix. Employing the Lagrangian multiplier $\tau$ and denoting $\xi_{i}$ as the $i$ th diagonal element of $\Xi$, the optimal solution is obtained as

$$
\left|\xi_{i}\right|^{2}=\frac{1}{\omega^{2} \Sigma_{i}}\left(\sqrt{\frac{\sum_{i} \omega_{i}^{2}\left(\sigma_{n_{\mathrm{d}_{2}}}^{2}+\sigma_{\mathrm{E}_{\mathrm{T}}}^{2} P_{\mathrm{R}}\right)}{\tau}}-\left(\sigma_{n_{\mathrm{d}_{2}}}^{2}+\sigma_{\mathrm{E}_{\mathrm{T}}}^{2} P_{\mathrm{R}}\right)\right)^{+},
$$

where $\Sigma_{i}$ signifies the $i$ th diagonal element of $\Sigma$ and $\tau$ indicates the water-level chosen to satisfy the power constraint.
3.3.2. Maximum Eigenvalue Constraint (Robust RD-MV). Using MVC, the optimization problem is similarly rewritten as

$$
\begin{array}{ll}
\min _{\mathbf{B}} & \operatorname{tr}\left(\mathrm{MSE}_{g}\right), \\
\text { s.t. } & \lambda_{\max }\left(\mathbf{B R}_{y} \mathbf{B}^{\dagger}\right)=L_{\mathrm{R}} .
\end{array}
$$

A similar approach as in the robust SRD-MV is used to compute the optimal precoder $\widehat{\mathbf{B}}=\overline{\mathbf{V}}_{\mathrm{g}} \boldsymbol{\Xi} \mathbf{V}_{\mathrm{h}}^{\dagger}$, where $\xi_{i}=\sqrt{L_{\mathrm{R}} / \Sigma_{i}}$.

\section{MIMO AF Relay with Direct Link}

The direct source-destination link is known to provide an increase in spatial diversity order in MIMO relay system, which is essential for improving performance, and thus should not be ignored $[29,30]$. It is assumed in this discussion that no channel state information (CSI) is allowed at the source, so it should be noted that all $\mathbf{F}$ terms in the derivation in this section are taken as $\mathbf{F}=\mathbf{I}_{N_{\mathrm{t}}}$, where $N_{\mathrm{t}}=N_{\mathrm{s}}$. Both relay and destination, however, have ICSI of all links. The input signal-to-noise ratio is given as $\sigma_{x}^{2} \triangleq P_{\mathrm{T}} / N_{\mathrm{t}}$.

4.1. Problem Formulation. The error vector is defined as $\mathbf{e} \triangleq \widetilde{\mathbf{y}}_{\mathrm{D}}-\mathbf{x}=\mathbf{W}_{\mathrm{d}}-\mathbf{x}$. Also, the corresponding error covariance or MSE matrix defined as a function of $\mathbf{W}$ and $\mathbf{Q}$ is denoted as $\mathbf{R}_{\mathrm{E}}(\mathbf{W}, \mathbf{Q}) \triangleq \varepsilon\left[\mathbf{e e}^{\dagger}\right]$.

Since CSIT at the source is not considered in this case, the optimization problem determines with global ICSI, the relay and destination filters, and is given as

$$
\begin{array}{ll}
\min _{\mathbf{W}, \mathbf{Q}} & \operatorname{tr}\left[\mathbf{R}_{\mathrm{E}}(\mathbf{W}, \mathbf{Q})\right] \\
\text { s.t. } & \operatorname{tr}\left(\mathbf{Q} \mathscr{A} \mathbf{Q}^{\dagger}\right) \leq P_{\mathbf{R}},
\end{array}
$$

where $P_{\mathrm{R}}$ represents the power constraint at the relay.

4.1.1. Optimum Destination Receiver. Although generally nonconvex, two subproblems for the optimization above can be considered where each problem is convex (or quasiconvex) with respect to $\mathbf{W}$ and $\mathbf{Q}$, respectively, given the other.

Theorem 4. As mentioned, the optimal destination filter is easily obtained for a given $\mathbf{Q}$ as

$$
\begin{aligned}
\widehat{\mathbf{W}}(\mathbf{Q})= & \left(\mathbf{R}_{\mathrm{T}}^{-1}+\widehat{\mathbf{H}}^{\dagger} \mathbf{Q}^{\dagger} \widehat{\mathbf{G}}^{\dagger} \mathbf{R}_{2}^{-1} \widehat{\mathbf{G}} \mathbf{Q} \widehat{\mathbf{H}}\right)^{-1} \\
& \times\left[\begin{array}{ll}
\widehat{\mathbf{T}}^{\dagger} \mathbf{R}_{1}^{-1} & \widehat{\mathbf{H}}^{\dagger} \mathbf{Q}^{\dagger} \widehat{\mathbf{G}}^{\dagger} \mathbf{R}_{2}^{-1}
\end{array}\right]
\end{aligned}
$$

where $\mathbf{R}_{\mathrm{T}}^{-1}=\sigma_{x}^{-2} \mathbf{I}_{N_{\mathrm{t}}}+\mathbf{T}^{\dagger} \mathbf{R}_{1}^{-1} \mathbf{T}$ and $\mathbf{R}_{1}=\sigma_{\mathrm{E}_{\mathrm{T}}}^{2} \operatorname{tr}\left(\sigma_{x}^{2} \mathbf{I}_{N_{\mathrm{t}}}\right) \mathbf{I}+\mathbf{R}_{n_{\mathrm{d}_{1}}}$, where $\mathbf{R}_{n_{\mathrm{d}_{1}}}$ is the covariance of the noise vector $\mathbf{n}_{\mathrm{d}_{1}}$. Here $\mathbf{R}_{2}=$ $\sigma_{\mathrm{E}_{\mathrm{G}}}^{2} \operatorname{tr}\left(\mathbf{Q} \Gamma \mathbf{Q}^{\dagger}\right) \mathbf{I}_{N_{\mathrm{d}}}+\sigma_{\mathrm{E}_{\mathrm{H}}}^{2} \operatorname{tr}\left(\sigma_{x}^{2} \mathbf{I}_{N_{\mathrm{t}}}\right) \widehat{\mathbf{G}} \mathbf{Q} \mathbf{Q}^{\dagger} \widehat{\mathbf{G}}^{\dagger}+\mathbf{R}_{n}$ and $\Gamma=$ $\sigma_{x}^{2} \hat{\mathbf{H}} \widehat{\mathbf{H}}^{\dagger}+\sigma_{\mathrm{E}_{\mathrm{H}}}^{2} \operatorname{tr}\left(\sigma_{x}^{2} \mathbf{I}_{N_{\mathrm{t}}}\right) \mathbf{I}_{N_{\mathrm{r}}}$. The covariance matrix of the effective noise vector during the second time slot is given by $\mathbf{R}_{n}=\sigma_{n_{\mathrm{r}}}^{2} \widehat{\mathbf{G}} \mathbf{Q} \mathbf{Q}^{\dagger} \widehat{\mathbf{G}}^{\dagger}+\left[\sigma_{n_{\mathrm{d}_{2}}}^{2}+\sigma_{\mathrm{E}_{\mathrm{G}}}^{2} \operatorname{tr}\left(\mathbf{Q} \sigma_{n_{\mathrm{r}}}^{2} \mathbf{Q}^{\dagger}\right)\right] \mathbf{I}_{N_{\mathrm{d}}}$. 
4.1.2. Error Covariance Matrix. The MSE matrix as a function of $\mathbf{Q}, \mathbf{R}_{\mathrm{E}}(\mathbf{Q})$, can be found by substituting the optimal receive filter into $\mathbf{R}_{\mathrm{E}}(\mathbf{W}, \mathbf{Q})$ and invoking matrix inversion lemma as follows:

$$
\mathbf{R}_{\mathrm{E}}(\mathbf{Q})=\left(\mathbf{R}_{\mathrm{T}}^{-1}+\widehat{\mathbf{H}}^{\dagger} \mathbf{Q}^{\dagger} \widehat{\mathbf{G}}^{\dagger} \mathbf{R}_{2}^{-1} \widehat{\mathbf{G}} \mathbf{Q} \widehat{\mathbf{H}}\right)^{-1} .
$$

Theorem 5. For a fixed $\mathbf{W}=\left[\begin{array}{ll}\mathbf{W}_{1} & \mathbf{W}_{2}\end{array}\right]$, the relay precoder $\mathbf{B}$ and receiver $\mathrm{L}_{\mathrm{R}}$, comprising the optimal relay transceiver $\widehat{\mathbf{Q}}=\mathbf{B L}_{\mathrm{R}}$, are calculated, respectively, as

$$
\begin{aligned}
\mathbf{B}= & \left\{\widehat{\mathbf{G}}^{\dagger} \mathbf{W}_{2}^{\dagger} \mathbf{W}_{2} \widehat{\mathbf{G}}+\mu \mathbf{I}_{N_{\mathrm{r}}}+\operatorname{tr}\left(\sigma_{\mathrm{E}_{\mathrm{T}}}^{2} \mathbf{W}_{2} \mathbf{W}_{2}^{\dagger}\right)\right\}^{-1} \widehat{\mathbf{G}}^{\dagger} \mathbf{W}_{2}^{\dagger}\left(\mathbf{I}-\mathbf{W}_{1} \widehat{\mathbf{T}}\right) \\
& \times\left\{\mathbf{I}_{N_{\mathrm{t}}}+\left[\widehat{\mathbf{H}}^{\dagger} \widehat{\mathbf{H}}+\frac{\sigma_{\mathrm{E}_{\mathrm{H}}}^{2}}{\sigma_{x}^{2}} \operatorname{tr}\left(\sigma_{x}^{2} \mathbf{I}_{N_{\mathrm{t}}}\right) \mathbf{I}_{N_{\mathrm{t}}}+\sigma_{x}^{-2} \mathbf{I}_{N_{\mathrm{t}}}\right]^{-1}\right. \\
& {\left.\left[\left(\sigma_{n_{\mathrm{r}}}^{2}+\sigma_{\mathrm{E}_{\mathrm{H}}}^{2} \operatorname{tr}\left(\sigma_{x}^{2} \mathbf{I}_{N_{\mathrm{t}}}\right)\right)\left(\sigma_{n_{\mathrm{d}}}^{2}+\left(\sigma_{\mathrm{E}_{\mathrm{T}}}^{2} \operatorname{tr}\left(\sigma_{x}^{2} \mathbf{I}_{N_{\mathrm{t}}}\right)\right)\right)^{-1} \widehat{\mathbf{T}}^{\dagger} \mathbf{T}\right]\right\}, } \\
\mathbf{L}_{\mathrm{R}}= & \left(\widehat{\mathbf{H}}^{\dagger} \widehat{\mathbf{H}}+\sigma_{\mathrm{E}_{\mathrm{H}}}^{2} \operatorname{tr}\left(\sigma_{x}^{2} \mathbf{I}_{N_{\mathrm{t}}}\right) \mathbf{R}_{\mathrm{T}}^{-1}+\mathbf{R}_{\mathrm{T}}^{-1}\right)^{-1} \widehat{\mathbf{H}}^{\dagger},
\end{aligned}
$$

where $\mathbf{B}$ is relay transmit Wiener filter for the second hop channel $\mathbf{G}, \mathbf{L}_{\mathrm{R}}$ is the relay receive Wiener filter $(R-W F)$ for the first hop channel, and $\mu$ is chosen to satisfy the relay constraint in (19).

\section{Proof. See Appendix D.}

When $\mathbf{T}$ is set to zero (i.e., when the direct link is negligible), the above equations result in AF relay scheme with no direct link above when the source precoder is not available at the source. It is also necessary to note that the considered robust transceiver optimization problem includes the problem under the perfect CSI assumption as a special case by setting channel errors to zeros.

4.1.3. Decomposition of $\mathbf{R}_{\mathrm{E}}$. As stated above, the error covariance matrix can be decomposed as a sum of two individual covariance matrices for the given optimal relay structure $\widehat{\mathbf{Q}}=\mathbf{B L}_{\mathrm{R}}$.

Theorem 6. The error covariance matrix is given as

$$
\begin{aligned}
\mathbf{R}_{\mathrm{E}}= & \left(\sigma_{n_{\mathrm{r}}}^{2}+\sigma_{\mathrm{E}_{\mathrm{H}}}^{2} \operatorname{tr}\left(\sigma_{x}^{2} \mathbf{I}_{N_{\mathrm{t}}}\right) \mathbf{I}_{N_{\mathrm{r}}}\right) \\
& \times\left(\widehat{\mathbf{H}}^{\dagger} \widehat{\mathbf{H}}+\mathbf{R}_{\mathrm{T}}^{-1}+\sigma_{\mathrm{E}_{\mathrm{H}}}^{2} \operatorname{tr}\left(\sigma_{x}^{2} \mathbf{I}_{N_{\mathrm{t}}}\right) \mathbf{R}_{\mathrm{T}}^{-1}\right)^{-1} \\
& +\left(\mathbf{\Omega}^{-1}+\mathbf{B}^{\dagger} \widehat{\mathbf{G}}^{\dagger} \mathbf{S}^{-1} \widehat{\mathbf{G}} \mathbf{B}\right)^{-1}
\end{aligned}
$$

where $\mathbf{S}=\left[\sigma_{n_{\mathrm{d}_{2}}}^{2}+\sigma_{\mathrm{E}_{\mathrm{G}}}^{2} \operatorname{tr}\left(\mathbf{B R}_{y} \mathbf{B}^{\dagger}\right)\right] \mathbf{I}_{N_{\mathrm{d}}}$ and $\mathbf{R}_{y}=\mathbf{L}_{\mathrm{R}} \mathscr{A} \mathbf{L}_{\mathrm{R}}^{\dagger}$. Also $\mathrm{L}_{\mathrm{R}}^{\dagger}$ is the covariance matrix of the relay receiver output signal, $\mathbf{L}_{\mathrm{R}} \mathbf{y}_{\mathrm{r}}$, and $\mathbf{\Omega} \in \mathbb{S}_{+}^{N_{\mathrm{t}}}$ is a positive semidefinite matrix defined as

$$
\boldsymbol{\Omega}=\mathbf{L}_{\mathrm{R}}\left[\sigma_{n_{\mathrm{r}}}^{2} \mathbf{I}_{N_{\mathrm{r}}}+\sigma_{\mathrm{E}_{\mathrm{H}}}^{2} \operatorname{tr}\left(\sigma_{x}^{2} \mathbf{I}_{N_{\mathrm{t}}}\right) \mathbf{I}_{N_{\mathrm{r}}}+\widehat{\mathbf{H}} \mathbf{R}_{\mathrm{T}} \widehat{\mathbf{H}}^{\dagger}\right] \mathbf{L}_{\mathrm{R}}^{\dagger}
$$

Proof. See Appendix E.

Since the first term in (23) is independent of $\mathbf{Q}$ or $\mathbf{W}$, only the second term needs to be optimized with respect to B. The joint optimization is the optimal relay precoder which is given as

$$
\begin{array}{ll}
\min _{\mathbf{B}} & \operatorname{tr}\left[\left(\mathbf{\Omega}^{-1}+\mathbf{B}^{\dagger} \widehat{\mathbf{G}}^{\dagger} \mathbf{S}^{-1} \widehat{\mathbf{G}} \mathbf{B}\right)\right] \\
\text { s.t. } & \operatorname{tr}\left(\mathbf{B R}_{y} \mathbf{B}^{\dagger}\right) \leq P_{\mathrm{R}} .
\end{array}
$$

4.2. Near-Optimal Relay Transceiver Design with Direct Link. The following eigenvalue decompositions are introduced to derive a new closed-form solution for the relay transceiver $\mathbf{Q}$ as follows:

$$
\begin{aligned}
\mathbf{G}^{\dagger} \mathbf{G} & =\mathbf{V}_{\mathrm{g}} \Lambda_{\mathrm{g}} \mathbf{V}_{\mathrm{g}}^{\dagger}, \\
\mathbf{\Omega} & =\mathbf{U}_{\mathrm{w}} \Lambda_{\mathrm{w}} \mathbf{U}_{\mathrm{w}}^{\dagger},
\end{aligned}
$$

where $\mathbf{V}_{\mathrm{g}} \in \mathbb{C}^{N_{\mathrm{r}} \times N_{\mathrm{r}}}$ and $\mathbf{U}_{\mathrm{w}} \in \mathbb{C}^{N_{\mathrm{t}} \times N_{\mathrm{t}}}$ are unitary matrices and $\Lambda_{\mathrm{g}}$ and $\boldsymbol{\Lambda}_{\mathrm{w}}$ are square matrices with descending eigenvalues as diagonal elements given by $\lambda_{\mathrm{g}, k} \in \mathbb{R}_{+}$for $k=$ $1, \ldots, N_{\mathrm{r}}$ and $\lambda_{\mathrm{w}, k} \in \mathbb{R}_{+}$for $k=1, \ldots, N_{\mathrm{t}}$, respectively. The optimal relay precoder $\mathbf{B}$ can be represented in a general form as

$$
\widehat{\mathbf{B}}=\overline{\mathbf{V}}_{\mathrm{g}} \boldsymbol{\Phi}_{1} \overline{\mathbf{U}}_{\mathrm{w}}^{\dagger}
$$

where $\overline{\mathbf{V}}_{\mathrm{g}} \in \mathbb{C}^{N_{\mathrm{r}} \times N_{\mathrm{t}}}$ denotes the first $N_{\mathrm{t}}$ columns of $\mathbf{V}_{\mathrm{g}}$. Substituting (27) into (25), the modified problem to determine optimal $\Phi_{1}$ is given as

$$
\begin{aligned}
\widehat{\boldsymbol{\Phi}}_{1} & =\arg \min f_{\mathrm{o}}\left(\boldsymbol{\Phi}_{1}\right), \\
\text { s.t. } f_{\mathrm{p}}\left(\boldsymbol{\Phi}_{1}\right) & \leq P_{\mathrm{R}},
\end{aligned}
$$

where $\quad f_{\mathrm{o}}\left(\boldsymbol{\Phi}_{1}\right)=\operatorname{tr}\left(\mathbf{S}\left(|\boldsymbol{\Phi}|^{2} \widetilde{\Lambda}_{\mathrm{g}}+\mathbf{S}\left(\widetilde{\Lambda}_{\mathrm{w}}\right)^{-1}\right)^{-1}\right), \quad f_{\mathrm{p}}\left(\boldsymbol{\Phi}_{1}\right)=$ $\operatorname{tr}\left(|\Phi|^{2} \mathbf{R}_{\mathrm{w}}\right), \widetilde{\Lambda}_{\mathrm{g}}$ is an $N_{\mathrm{t}} \times N_{\mathrm{t}}$ upper left submatrix of $\boldsymbol{\Lambda}_{\mathrm{g}}$, and $\mathbf{R}_{\mathrm{w}}=\overline{\mathbf{U}}_{\mathrm{w}}^{\dagger} \mathbf{R}_{\mathrm{y}} \overline{\mathbf{U}}_{\mathrm{w}}^{\mathrm{g}}$.

Diagonalizing the terms simultaneously inside the trace in (28) results in the optimal solution for minimizing the trace. However, due to the nondiagonal structure of $\mathbf{R}_{\mathrm{w}}$ and the nonconvex nature of the problem, there is no such case for all $\Phi_{1}$, unlike the case with no direct link. A structural constraint is imposed on $\boldsymbol{\Phi}_{1}$ so that a diagonal matrix defined as $\boldsymbol{\Phi}_{\mathrm{d}} \in \mathbb{C}^{N_{\mathrm{t}} \times N_{\mathrm{t}}}$ with diagonal entries $\phi_{1}, \ldots, \phi_{N_{t}}$ can be found, where $f_{\mathrm{o}}\left(\boldsymbol{\Phi}_{\mathrm{d}}\right)=f_{\mathrm{o}}\left(\widehat{\boldsymbol{\Phi}}_{1}\right)$. This will facilitate finding a closed-form solution. Though $\Phi_{\mathrm{d}}$ is suboptimal because it may increase the power consumption, that is, $f_{\mathrm{p}}\left(\boldsymbol{\Phi}_{\mathrm{d}}\right) \leq f_{\mathrm{p}}\left(\widehat{\boldsymbol{\Phi}}_{1}\right)$ with the same MSE, $\boldsymbol{\Phi}_{\mathrm{d}}$ is considered as a solution as it provides a diagonal structure and thus a simple convex problem and moreover lower complexity. We thus attain the following optimization problem:

$$
\begin{aligned}
\widehat{\boldsymbol{\Phi}}_{\mathrm{d}} & =\arg \min f_{\mathrm{o}}\left(\boldsymbol{\Phi}_{\mathrm{d}}\right), \\
\text { s.t. } f_{\mathrm{p}}\left(\boldsymbol{\Phi}_{\mathrm{d}}\right) & \leq P_{\mathrm{R}} .
\end{aligned}
$$

The solution for (29) is found by using the Lagrangian multiplier $\mu$.

Using definitions for $\mathbf{B}, \mathbf{R}_{\mathrm{w}}$, and $\mathbf{G}^{\dagger} \mathbf{G}$ above, and noting that 


$$
\mathbf{S}=S \mathbf{I}_{N_{\mathrm{d}}}=\left[\sigma_{n_{\mathrm{d}_{2}}}^{2}+\sigma_{\mathrm{E}_{\mathrm{G}}}^{2} \operatorname{tr}\left(\mathbf{B R}_{\mathrm{y}} \mathbf{B}^{\dagger}\right)\right] \mathbf{I}_{N_{\mathrm{d}}}=\left(\sigma_{n_{\mathrm{d}_{2}}}^{2}+\sigma_{\mathrm{E}_{\mathrm{G}}}^{2} P_{\mathrm{R}}\right) \mathbf{I}_{N_{\mathrm{d}}} .
$$

The minimization problem can be rewritten as

$$
\begin{array}{ll}
\min _{\mathbf{B}} \operatorname{tr}\left[\left(\boldsymbol{\Omega}^{-1}+\mathbf{B}^{\dagger} \widehat{\mathbf{G}}^{\dagger} \mathbf{S}^{-1} \widehat{\mathbf{G}} \mathbf{B}\right)^{-1}\right] \\
=\min _{\mathbf{B}} \operatorname{tr}\left[\mathbf{S}\left(\mathbf{B}^{\dagger} \mathbf{G}^{\dagger} \widehat{\mathbf{G}} \mathbf{B}+\mathbf{S} \boldsymbol{\Omega}^{-1}\right)^{-1}\right] \\
\text { s.t. } & \operatorname{tr}\left(\left|\boldsymbol{\Phi}_{k}\right|^{2} \mathbf{R}_{\mathrm{w}}\right) \leq P_{\mathrm{R}} .
\end{array}
$$

Each element of $\widehat{\Phi}_{\mathrm{d}}$ is determined as

$$
\left|\widehat{\phi}_{k}\right|^{2}=\frac{1}{\lambda_{\mathrm{g}, k} \lambda_{\mathrm{w}, k}}\left(\sqrt{\frac{\left(\sigma_{n_{\mathrm{d}_{2}}}^{2}+\sigma_{\mathrm{E}_{\mathrm{G}}}^{2} P_{\mathrm{R}}\right) \lambda_{\mathrm{g}, k} \lambda_{\mathrm{w}, k}^{2}}{\mu r_{k}}}-\left(\sigma_{n_{\mathrm{d}_{2}}}^{2}+\sigma_{\mathrm{E}_{\mathrm{G}}}^{2} P_{\mathrm{R}}\right)\right)^{+},
$$

where $k=1, \ldots, N_{\mathrm{t}}$. Here, $r_{k}$ designates the $k$ th diagonal element of $\mathbf{R}_{\mathrm{w}}$, and $\mu$ is chosen to satisfy the power constraint in (29). It is worthwhile to note that the derived solution is globally optimal, that is, $\widehat{\boldsymbol{\Phi}}_{1}=\widehat{\boldsymbol{\Phi}}_{\mathrm{d}}$ when $\mathbf{R}_{\mathrm{w}}$ is a diagonal matrix. The closed form for the relay transceiver as stated above is thus given by $\mathbf{Q}=\widehat{\mathbf{B}} \mathbf{L}_{\mathrm{R}}=\overline{\mathbf{V}}_{\mathrm{g}} \widehat{\boldsymbol{\Phi}}_{\mathrm{d}} \overline{\mathbf{U}}_{\mathrm{w}}^{\dagger} \mathbf{L}_{\mathrm{R}}$.

\section{Simulation Results}

To support the derivations and also to illustrate the effect of the ICSI on the system performance, simulation results are presented in this section. The notation $N_{\mathrm{t}} \times N_{\mathrm{r}} \times N_{\mathrm{d}}$ is used to denote the system with $N_{\mathrm{t}}$ source, $N_{\mathrm{r}}$ relay, and $N_{\mathrm{d}}$ destination antennas. $N_{\mathrm{s}}$ is used to denote the number of data streams. For both cases, it is assumed that the channel estimation error variances are equal, that is, $\sigma_{\mathrm{E}_{\mathrm{H}}}^{2}=\sigma_{\mathrm{E}_{\mathrm{T}}}^{2}=$ $\sigma_{\mathrm{E}_{\mathrm{G}}}^{2}=\sigma_{\mathrm{E}}^{2}$.

5.1. AF Scheme without Direct Link Results. Monte Carlo simulations are performed to illustrate the BER and MSE performance of the proposed schemes for source-to-relayto-destination (SRD) relaying system with negligible direct link. The proposed designs using norm power constraint (NC) and maximum eigenvalue (MV) constraints are compared with the traditional naive AF scheme where only a power normalizing operation is performed at the relay, that is, $\mathbf{Q}=\sqrt{P_{\mathrm{R}} / \operatorname{tr}\left(\sigma_{\mathrm{x}}^{2} \mathbf{H} \mathbf{F F}^{\dagger} \mathbf{H}^{\dagger}+\sigma_{n_{\mathrm{r}}}^{2} \mathbf{I}_{N_{\mathrm{r}}}\right)} \mathbf{I}_{N_{\mathrm{r}}}$. Our schemes are also compared with the case of perfect CSI discussed in [2], which corresponds to setting all channel error terms to zero, that is, $\sigma_{\mathrm{E}}^{2}=0$. We assume that the noise covariance $\sigma_{n_{\mathrm{r}}}^{2}=\sigma_{n_{\mathrm{d} 2}}^{2}=1$ and $N_{\mathrm{r}}=4$. The total signal-to-noise ratio $\left(\mathrm{SNR}_{0}\right)$ is defined as $P / \sigma_{n}^{2}$ with $P_{\mathrm{T}}=P_{\mathrm{R}}=P / 2 . \mathrm{SNR}_{1} \triangleq$ $P_{\mathrm{T}} /\left(N_{\mathrm{s}} \sigma_{n_{\mathrm{r}}}^{2}\right)$ indicates the SNR of the source-to-relay link and that between the relay-to-destination link is $\mathrm{SNR}_{2} \triangleq$ $P_{\mathrm{R}} /\left(N_{\mathrm{s}} \sigma_{n_{\mathrm{d}}}^{2}\right)$, respectively. Also, the peaks are set to $L_{\mathrm{T}}=$ $P_{\mathrm{T}} / N_{\mathrm{s}}$ and $L_{\mathrm{R}}=P_{\mathrm{R}} / N_{\mathrm{s}}$ in order to satisfy the peak power constraints. It is assumed that all channel matrices are independent and identically distributed (i.i.d.) complex Gaussian with mean zero and unit variance.

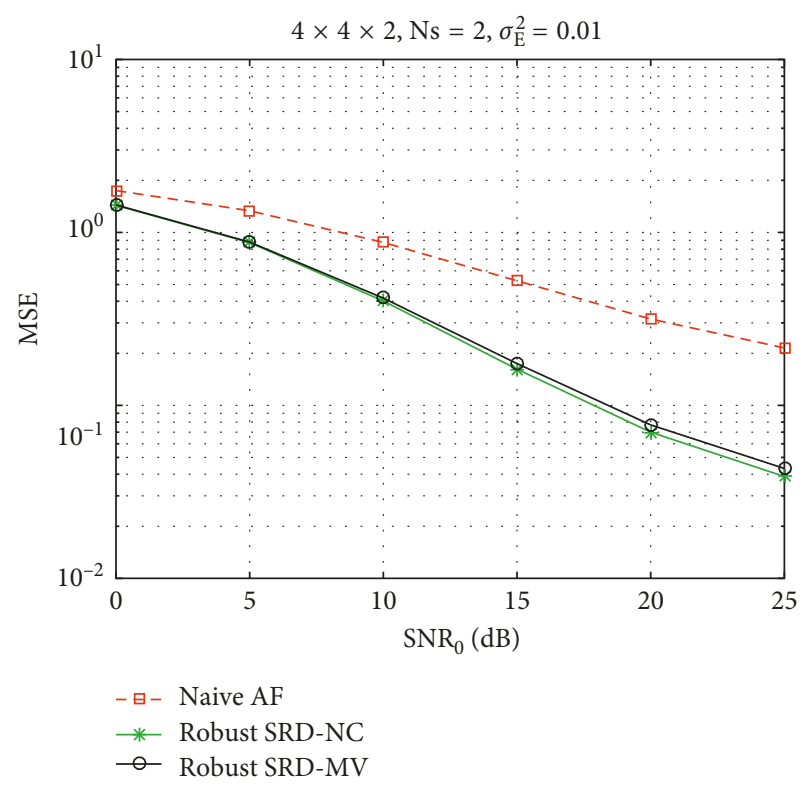

FIGURE 2: MSE performance comparison of proposed designs and naive $\mathrm{AF}$ as a function of $\mathrm{SNR}_{0}$.

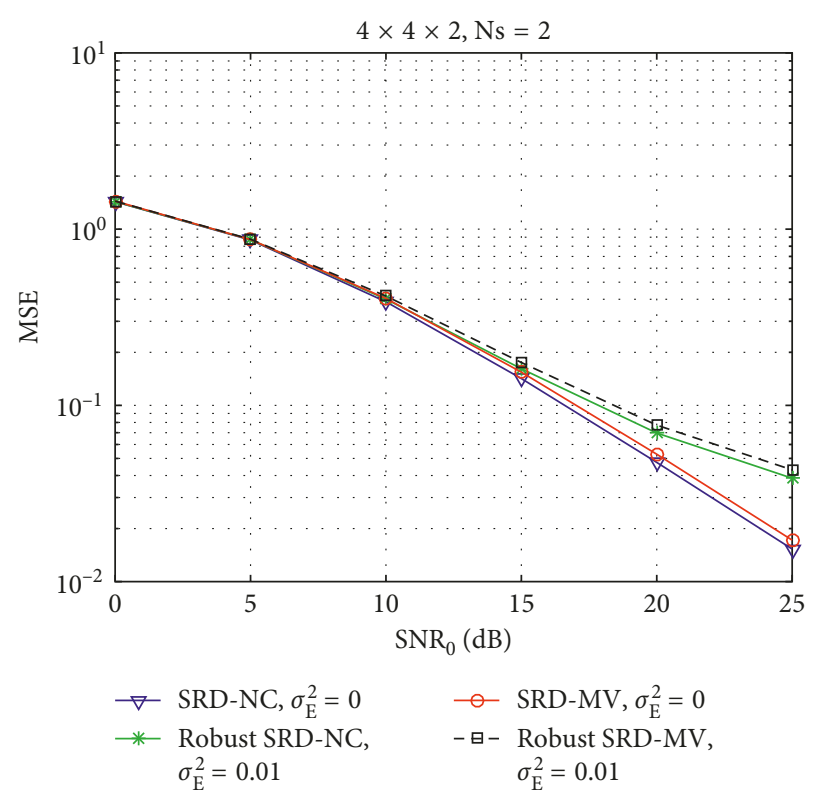

FIGURE 3: MSE performance of various designs as a function of $\mathrm{SNR}_{0}$.

Figure 2 shows the MSE performance of an $4 \times 4 \times 2$, $N_{\mathrm{s}}=2$ system. Here, an improved performance of the proposed schemes is shown compared with the naive AF relaying scheme in terms of the MSE. This is because according to [31], naive AF does not consider CSI error. In Figure 3, the deteriorating effects on performance caused by errors in channel estimation is shown by comparing the MSE performance in a system with perfect CSI $\left(\sigma_{\mathrm{E}}^{2}=0\right)$ and that of a system with ICSI using proposed schemes. As expected, both schemes with perfect CSI have the lowest MSE and thus outperform the schemes with ICSI. 


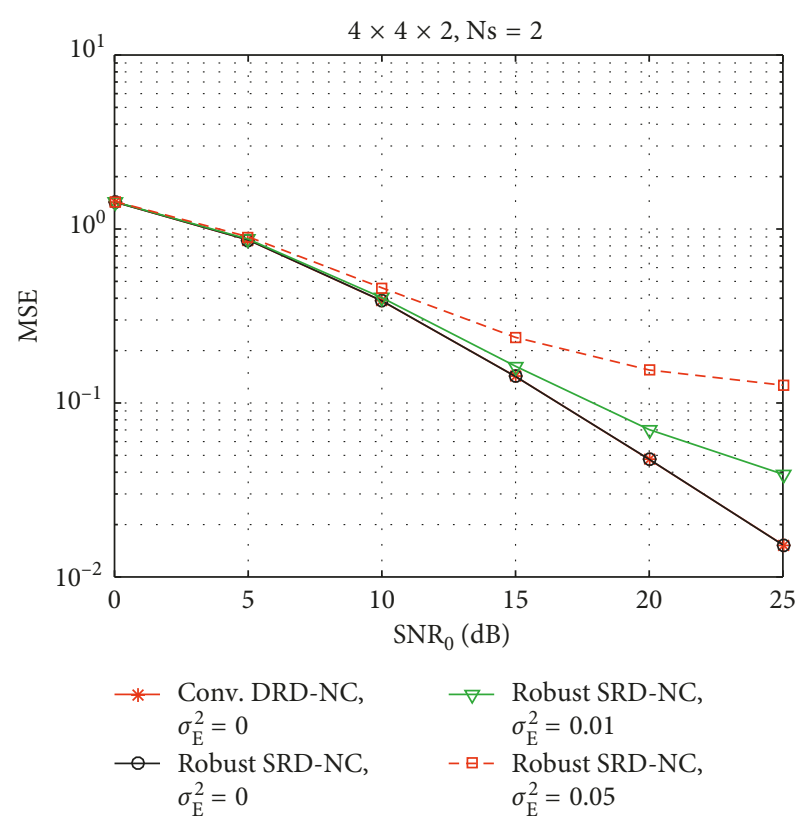

FIGURE 4: MSE performance for SRD-NC scheme as a function of $\mathrm{SNR}_{0}$ for different error values.

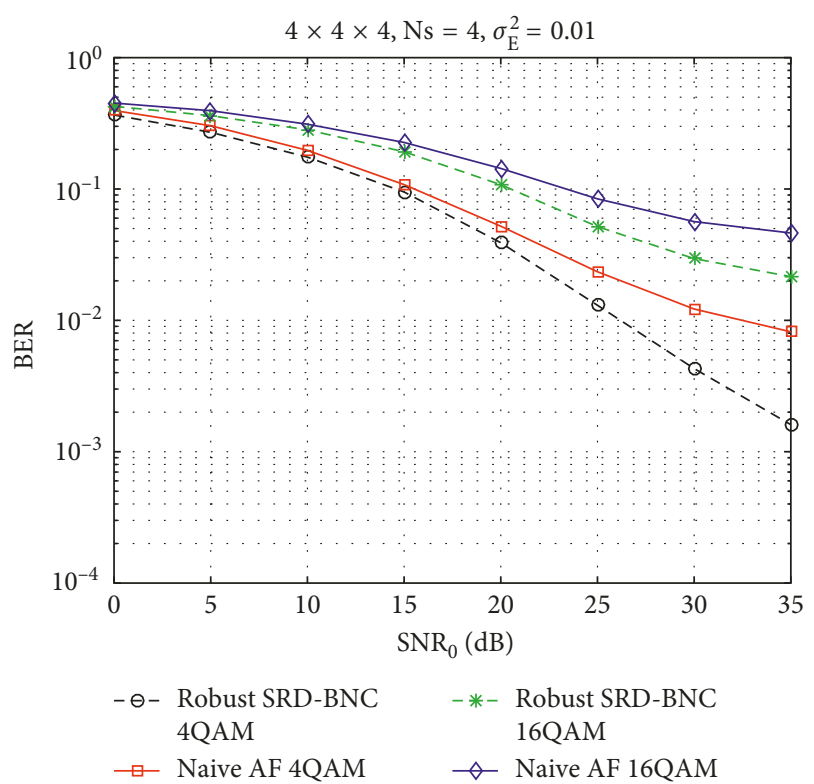

FIGURE 5: BER performance of various designs as a function of $\mathrm{SNR}_{0}$ for different constellations.

Figure 4 further shows that increasing channel estimation errors increasingly degrades the performance of MSE as the transceiver design is misled in the presence of ICSI, and it also confirms the fact that setting errors to zero gives the same results in [32]. The BER performance of SRD relaying systems with $N_{\mathrm{t}}=N_{\mathrm{r}}=N_{\mathrm{d}}=N_{\mathrm{s}}=4$ antennas for the proposed scheme is compared with the naive AF scheme for different constellations. Similarly, the proposed scheme outperforms the naive AF scheme as shown in Figure 5.

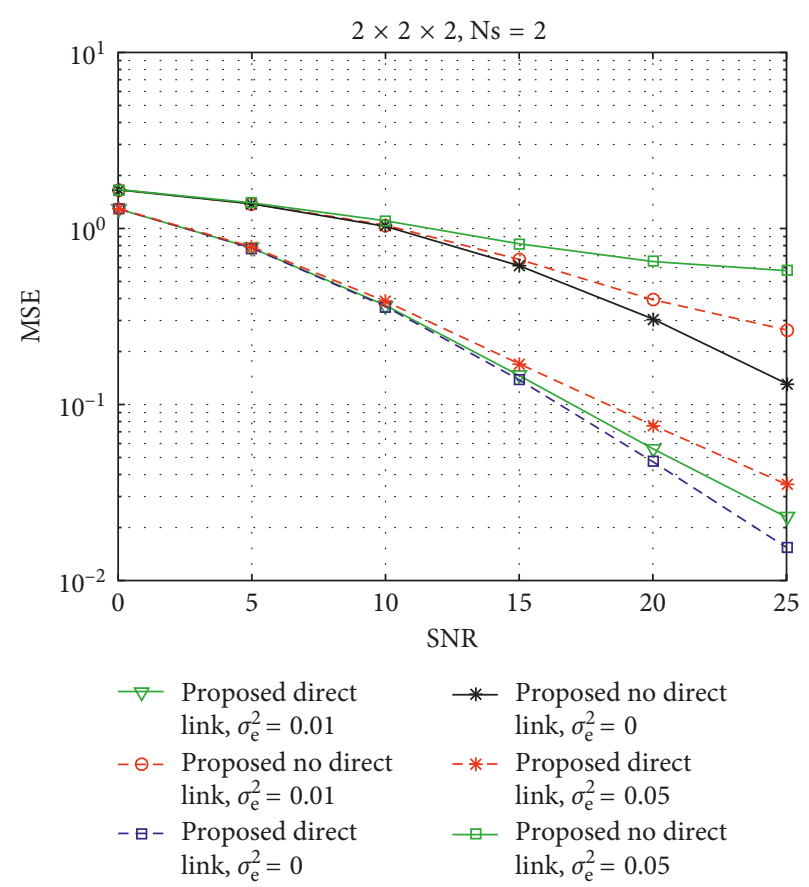

FIGURE 6: Comparing MSE performance of system with direct link and no direct link as a function of $\mathrm{SNR}_{0}$.

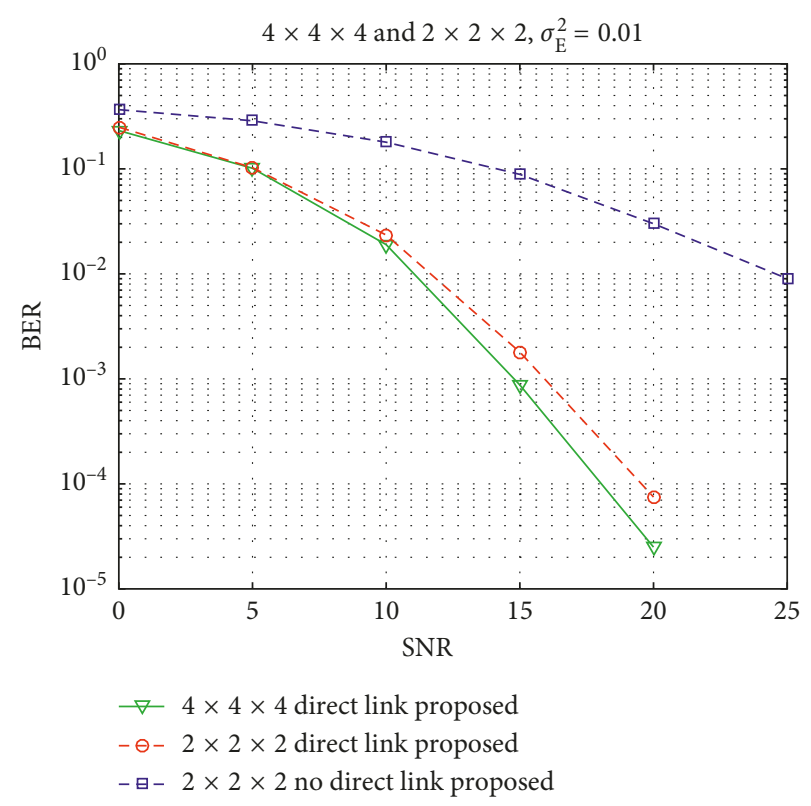

FIGURE 7: BER performance of system with direct link and no direct link as a function of $\mathrm{SNR}_{0}$.

5.2. AF Scheme with Direct Link. Figure 6 compares the proposed schemes with direct link and no direct link. It confirms the importance of including the direct link in our proposed designs. Further, the proposed scheme, even with no CSI at the source, performs better than when the direct link is not considered even with an optimized source precoder. Also, the benefit of including the direct link is affirmed by the plot in Figure 7 by considering different number of antennas. 


\section{Conclusion}

The problem of ICSI in linear nonregenerative MIMO relay communications using MMSE criterion has been addressed. The closed-form solutions for the optimal source and relay precoder are derived to minimize the MMSE of symbol estimations. We presented a detailed modeling of imperfect channel estimation at all nodes in the MIMO system. It is also shown that, though nonconvex in nature, applying a structural constraint allows a diagonal structure that results in a simple convex problem making solution of optimization problem easier. Also, it is shown that the robust designs proposed include the perfect CSI case when the channel errors are set to zero. Simulation results show an improved robustness of the proposed algorithm against CSI errors.

Results further demonstrate a much better performance of the robust schemes in terms of both BER and MSE as compared with other relaying strategies. At various levels of CSI mismatch, proposed algorithms further enhance performance with respect to the pure relaying systems. This confirms the importance of including the direct link in our designs.

\section{Appendix}

\section{A. Proof of Theorem 1}

To find the optimal receive filter, the MSE equation is first obtained. The MSE as a function of the receive filter and relay transceiver is given by

$$
\begin{aligned}
\operatorname{MSE}(\mathbf{Q}, \mathbf{W})= & \varepsilon\left[\operatorname{tr}\left(\mathbf{W}_{\mathrm{D}} \hat{\mathbf{y}}_{\mathrm{D}}-\mathbf{x}\right)\left(\mathbf{W}_{\mathrm{D}} \dot{\mathbf{y}}_{\mathrm{D}}-\mathbf{x}\right)^{\dagger}\right], \\
= & \varepsilon\left\|\gamma^{-1} \mathbf{W}_{\mathrm{D}}\left[\left(\mathbf{G} \mathbf{Q} \mathbf{H} \mathbf{F}-\mathbf{I}_{N_{\mathrm{s}}}\right) \mathbf{x}+\mathbf{G} \mathbf{Q} \mathbf{n}_{\mathrm{r}}+\mathbf{n}_{\mathrm{d}_{2}}\right]\right\|^{2}, \\
= & \varepsilon_{\mathrm{E}_{\mathrm{H}}, \mathrm{E}_{\mathrm{T}}}\left\{\operatorname{tr}\left[\gamma^{-2} \sigma_{x}^{2}\left(\mathbf{W}_{\mathrm{D}} \mathbf{G} \mathbf{Q H F}\right)\left(\mathbf{W}_{\mathrm{D}} \mathbf{G Q H F}\right)^{\dagger}\right]\right\} \\
& -\operatorname{tr}\left(\gamma^{-1} \sigma_{x}^{2} \mathbf{W}_{\mathrm{D}} \widehat{\mathbf{G}} \mathbf{Q} \widehat{\mathbf{H}} \mathbf{F}\right)-\operatorname{tr}\left(\gamma^{-1} \sigma_{x}^{2} \mathbf{W}_{\mathrm{D}} \widehat{\mathbf{G}} \mathbf{Q} \widehat{\mathbf{H}} \mathbf{F}\right)^{\dagger} \\
& +\operatorname{tr}\left(\mathbf{R}_{x}\right)+\operatorname{tr}\left[\gamma^{-2} \mathbf{W}_{\mathrm{D}}\left(\varepsilon_{\mathrm{E}_{\mathrm{T}}}\left(\mathbf{G} \mathbf{Q} \sigma_{n_{\mathrm{r}}}^{2} \mathbf{Q}^{\dagger} \mathbf{G}\right)^{\dagger}\right) \mathbf{W}_{\mathrm{D}}^{\dagger}\right] \\
& +\operatorname{tr}\left(\gamma^{-2} \mathbf{W}_{\mathrm{D}} \sigma_{n_{\mathrm{d}_{2}}}^{2} \mathbf{W}_{\mathrm{D}}^{\dagger}\right) .
\end{aligned}
$$

The channel error terms $E_{\mathrm{H}}$ and $E_{\mathrm{T}}$ are independent, and thus the first term in (A.1) can be found as

$$
\begin{aligned}
& a=\varepsilon_{\mathrm{E}_{\mathrm{H}}, \mathrm{E}_{\mathrm{T}}}\left[\operatorname{tr}\left(\gamma^{-2} \sigma_{x}^{2}\left(\mathbf{W}_{\mathrm{D}} \mathbf{G Q H F}\right)\left(\mathbf{W}_{\mathrm{D}} \mathbf{G Q H F}\right)^{\dagger}\right)\right], \\
& =\operatorname{tr}\left[\gamma^{-2} \sigma_{x}^{2} \mathbf{W}_{\mathrm{D}} \varepsilon_{\mathrm{E}_{\mathrm{T}}}\left(\mathbf{G} \mathbf{Q} \varepsilon_{\mathrm{E}_{\mathrm{H}}}\left(\sigma_{x}^{2} \mathbf{H F F H}^{\dagger}\right) \mathbf{Q}^{\dagger} \mathbf{G}^{\dagger}\right) \mathbf{W}_{\mathrm{D}}^{\dagger}\right] \text {. }
\end{aligned}
$$

Knowing $\quad \mathbf{H} \triangleq \widehat{\mathbf{H}}+\mathbf{E}_{\mathrm{H}}, \quad \varepsilon_{\mathrm{E}_{\mathrm{H}}}\left(\sigma_{x}^{2} \mathbf{H} \mathbf{F} \mathbf{F}^{\dagger} \mathbf{H}^{\dagger}\right) \quad$ yields $\quad \Gamma=$ $\sigma_{x}^{2} \hat{\mathbf{H}} \mathbf{F} \mathbf{F}^{\dagger} \widehat{\mathbf{H}}^{\dagger}+\sigma_{\mathrm{E}_{\mathrm{H}}}^{2} \operatorname{tr}\left(\sigma_{x}^{2} \mathbf{F F}^{\dagger}\right) \mathbf{I}_{N_{r}}$. Thus, we get $a=$ $\operatorname{tr}\left[\gamma^{-2} \mathbf{W}_{\mathrm{D}} \mathcal{E}_{\mathrm{E}_{\mathrm{T}}}\left(\mathbf{G} \mathbf{Q} \Gamma \mathbf{Q}^{\dagger} \mathbf{G}^{\dagger}\right) \mathbf{W}_{\mathrm{D}}^{\dagger}\right]$. Similarly, after some manipulations, it becomes

$$
\begin{aligned}
a= & \operatorname{tr}\left[\gamma ^ { - 2 } \left(\mathbf{W}_{\mathrm{D}} \widehat{\mathbf{G}} \mathbf{Q} \Gamma \mathbf{Q}^{\dagger} \widehat{\mathbf{G}}^{\dagger} \mathbf{W}_{\mathrm{D}}^{\dagger}\right.\right. \\
& \left.\left.+\sigma_{\mathrm{E}_{\mathrm{T}}}^{2} \operatorname{tr}\left(\mathbf{W}_{\mathrm{D}} \mathbf{W}_{\mathrm{D}}^{\dagger}\right) \operatorname{tr}\left(\mathbf{Q} \Gamma \mathbf{Q}^{\dagger}\right) \mathbf{I}_{N_{\mathrm{d}}}\right)\right] .
\end{aligned}
$$

As a result, the MSE is written as
$\operatorname{MSE}(\mathbf{Q}, \mathbf{W})$

$$
\begin{aligned}
= & \operatorname{tr}\left[\gamma^{-2} \mathbf{W}_{\mathrm{D}}\left(\widehat{\mathbf{G}} \mathbf{Q} \mathscr{A} \mathbf{Q}^{\dagger} \widehat{\mathbf{G}}^{\dagger}+\sigma_{\mathrm{E}_{\mathrm{T}}}^{2} \operatorname{tr}\left(\mathbf{Q} \mathscr{A} \mathbf{Q}^{\dagger}\right)+\sigma_{n_{\mathrm{d}_{2}}}^{2}\right) \mathbf{W}_{\mathrm{D}}^{\dagger}\right] \\
& -\operatorname{tr}\left(\gamma^{-1} \sigma_{x}^{2} \mathbf{W}_{\mathrm{D}} \widehat{\mathbf{G}} \mathbf{Q} \widehat{\mathbf{H}} \mathbf{F}\right)-\operatorname{tr}\left(\gamma^{-1} \sigma_{x}^{2} \mathbf{W}_{\mathrm{D}} \widehat{\mathbf{G}} \mathbf{Q} \widehat{\mathbf{H}} \mathbf{F}\right)^{\dagger}+\operatorname{tr}\left(\mathbf{R}_{x}\right), \\
= & \operatorname{tr}\left[\gamma^{-2} \mathbf{W}_{\mathrm{D}}\left(\widehat{\mathbf{G}} \mathbf{Q} \mathbf{T} \mathbf{Q}^{\dagger} \widehat{\mathbf{G}}^{\dagger}+\beta \mathbf{I}_{N_{\mathrm{d}}}\right) \mathbf{W}_{\mathrm{D}}^{\dagger}\right] \\
& -\operatorname{tr}\left(\gamma^{-1} \sigma_{x}^{2} \mathbf{W}_{\mathrm{D}} \widehat{\mathbf{G}} \mathbf{Q} \widehat{\mathbf{H}} \mathbf{F}\right)-\operatorname{tr}\left(\gamma^{-1} \mathbf{W}_{\mathrm{D}} \widehat{\mathbf{G}} \mathbf{Q} \widehat{\mathbf{H}} \mathbf{F} \mathbf{R}_{x}\right)^{\dagger}+\operatorname{tr}\left(\mathbf{R}_{x}\right),
\end{aligned}
$$

where $\mathscr{A} \triangleq \sigma_{x}^{2} \widehat{\mathbf{H}} \mathbf{F F}^{\dagger} \widehat{\mathbf{H}}^{\dagger}+\left[\sigma_{n_{\mathrm{r}}}^{2}+\sigma_{\mathrm{E}_{\mathrm{H}}}^{2} \operatorname{tr}\left(\sigma_{x}^{2} \mathbf{F F}^{\dagger}\right)\right] \mathbf{I}_{N_{\mathrm{r}}}$

The MSE is then differentiated with respect to $\mathbf{W}$ and set to zero as follows:

$$
\begin{aligned}
\frac{\partial}{\partial \mathbf{W}^{\dagger}} & \operatorname{MSE}(\mathbf{Q}, \mathbf{W}) \\
= & \frac{\partial}{\partial \mathbf{W}^{\dagger}}\left\{\operatorname{tr}\left(\gamma^{-2} \mathbf{W}_{\mathrm{D}}\left[\widehat{\mathbf{G}} \mathbf{Q} \mathscr{A} \mathbf{Q}^{\dagger} \widehat{\mathbf{G}}^{\dagger}+\beta \mathbf{I}_{N_{\mathrm{d}}}\right] \mathbf{W}_{\mathrm{D}}^{\dagger}\right)\right. \\
& -\operatorname{tr}\left(\gamma^{-1} \sigma_{x}^{2} \mathbf{W}_{\mathrm{D}} \widehat{\mathbf{G}} \mathbf{Q} \widehat{\mathbf{H}} \mathbf{F}\right)-\operatorname{tr}\left(\gamma^{-1} \sigma_{x}^{2} \mathbf{W}_{\mathrm{D}} \widehat{\mathbf{G}} \mathbf{Q} \widehat{\mathbf{H}} \mathbf{F}\right)^{\dagger} \\
& \left.+\operatorname{tr}\left(\mathbf{R}_{x}\right)\right\}=0 .
\end{aligned}
$$

It becomes $2 \gamma^{-2} \widetilde{W}_{\mathrm{D}}\left(\widehat{\mathbf{G}} \mathbf{Q} \mathscr{A} \mathbf{Q}^{\dagger} \widehat{\mathbf{G}}^{\dagger}+\beta \mathbf{I}_{N_{\mathrm{d}}}\right)-2 \gamma^{-1} \sigma_{x}^{2}$ $\left(\mathbf{F}^{\dagger} \widehat{\mathbf{H}}^{\dagger} \mathbf{Q}^{\dagger} \widehat{\mathbf{G}}^{\dagger}\right)=0$. Finally, the optimal filter is obtained by $\widehat{\mathbf{W}}_{\mathrm{D}}=\gamma \sigma_{x}^{2}\left(\mathbf{F}^{\dagger} \widehat{\mathbf{H}}^{\dagger} \mathbf{Q}^{\dagger} \widehat{\mathbf{G}}^{\dagger}\right)\left(\widehat{\mathbf{G}} \mathbf{Q} \mathscr{A} \mathbf{Q}^{\dagger} \widehat{\mathbf{G}}^{\dagger}+\beta \mathbf{I}_{N_{\mathrm{d}}}\right)^{-1}$.

\section{B. Proof of Theorem 2}

To derive the optimal relay transceiver, the derivative of the cost function in (9) is set to zero as follows:

$$
\begin{aligned}
\frac{\partial \mathscr{C}}{\partial \mathbf{Q}^{*}}= & \left(\gamma^{-2} \widehat{\mathbf{G}}^{\dagger} \mathbf{W}_{\mathrm{D}}^{\dagger} \mathbf{W}_{\mathrm{D}} \widehat{\mathbf{G}} \mathbf{Q}+\lambda \mathbf{Q}\right) \mathscr{A} \\
& +\operatorname{tr}\left(\gamma^{-2} \sigma_{\mathrm{E}_{\mathrm{T}}^{2}} \mathbf{W}_{\mathrm{D}} \mathbf{W}_{\mathrm{D}}^{\dagger}\right) \mathbf{Q} \mathscr{A}-\gamma^{-1} \sigma_{x}^{2} \widehat{\mathbf{G}}^{\dagger} \mathbf{W}_{\mathrm{D}}^{\dagger} \mathbf{F}^{\dagger} \widehat{\mathbf{H}}^{\dagger}=0, \\
\frac{\partial \mathscr{C}}{\partial \gamma}= & \operatorname{tr}\left(\mathbf{W}_{\mathrm{D}} \widehat{\mathbf{G}} \mathbf{Q} \mathscr{A} \mathbf{Q}^{\dagger} \widehat{\mathbf{G}}^{\dagger} \mathbf{W}_{\mathrm{D}}^{\dagger}+\sigma_{n_{\mathrm{d}_{2}}}^{2} \mathbf{W}_{\mathrm{D}} \mathbf{W}_{\mathrm{D}}^{\dagger}\right) \\
& -\operatorname{tr}\left(\sigma_{\mathrm{E}_{\mathrm{T}}}^{2} \mathbf{W}_{\mathrm{D}} \mathbf{W}_{\mathrm{D}}^{\dagger}\right) \operatorname{tr}\left(\mathbf{Q} \mathscr{A} \mathbf{Q}^{\dagger}\right)-\gamma \sigma_{x}^{2} \mathbf{W}_{\mathrm{D}} \widehat{\mathbf{G}} \mathbf{Q} \widehat{\mathbf{H}} \mathbf{F}=0 .
\end{aligned}
$$

Combining the power constraint in (8) and (A.5), we get $\widehat{\mathbf{Q}}=\gamma \widetilde{\mathbf{Q}}$, where

$$
\begin{aligned}
\widetilde{\mathbf{Q}}= & \gamma\left[\widehat{\mathbf{G}}^{\dagger} \mathbf{W}_{\mathrm{D}}^{\dagger} \mathbf{W}_{\mathrm{D}} \widehat{\mathbf{G}}+\lambda \gamma^{2} \mathbf{I}_{N_{\mathrm{r}}}+\operatorname{tr}\left(\sigma_{\mathrm{E}_{\mathrm{T}}}^{2} \mathbf{W}_{\mathrm{D}} \mathbf{W}_{\mathrm{D}}^{\dagger}\right) \mathbf{I}_{N_{\mathrm{r}}}\right]^{-1} \\
& \times \sigma_{x}^{2} \widehat{\mathbf{G}}^{\dagger} \mathbf{W}_{\mathrm{D}}^{\dagger} \mathbf{F}^{\dagger} \widehat{\mathbf{H}}^{\dagger} \mathscr{A}^{-1},
\end{aligned}
$$

here $\mu=\lambda \gamma^{2}$ and $\gamma=\sqrt{P_{\mathrm{R}} / \operatorname{tr}\left(\widetilde{\mathbf{Q}} \mathscr{A} \widetilde{\mathbf{Q}}^{\dagger}\right)}$. Also, defining $\mathbf{A}$ and $\mathbf{D}$ as $\mathbf{A} \triangleq\left[\widehat{\mathbf{G}}^{\dagger} \mathbf{W}_{\mathrm{D}}^{\dagger} \mathbf{W}_{\mathrm{D}} \widehat{\mathbf{G}}+\mu \mathbf{I}_{N_{\mathrm{r}}}+\operatorname{tr}\left(\sigma_{\mathrm{E}_{\mathrm{T}}}^{2} \mathbf{W}_{\mathrm{D}} \mathbf{W}_{\mathrm{D}}^{\dagger}\right) \mathbf{I}_{N_{\mathrm{r}}}\right]^{-1}$ and $\mathbf{D} \triangleq \mathscr{A}^{-1}$, respectively, we get $\widetilde{\mathbf{Q}}=\gamma \mathbf{A} \sigma_{x}^{2} \widehat{\mathbf{G}}^{\dagger} \mathbf{W}_{\mathrm{D}}^{\dagger} \mathbf{F}^{\dagger} \widehat{\mathbf{H}}^{\dagger} \mathbf{D}$. 
The last term of (B.1) can be modified as

$$
\begin{aligned}
\operatorname{tr}\left(\gamma \sigma_{x}^{2} \mathbf{W}_{\mathrm{D}} \widehat{\mathbf{G}} \widehat{\mathbf{Q}} \widehat{\mathbf{H}} \mathbf{F}\right)= & \operatorname{tr}\left\{\gamma ^ { 2 } \left[\widehat{\mathbf{G}}^{\dagger} \mathbf{W}_{\mathrm{D}}^{\dagger} \mathbf{W}_{\mathrm{D}} \widehat{\mathbf{G}}+\mu \mathbf{I}_{N_{\mathrm{r}}}\right.\right. \\
& \left.\left.+\operatorname{tr}\left(\sigma_{\mathrm{E}_{\mathrm{T}}^{2}}^{2} \mathbf{W}_{\mathrm{D}} \mathbf{W}_{\mathrm{D}}^{\dagger}\right) \mathbf{I}_{N_{\mathrm{r}}}\right] \widetilde{\mathbf{Q}} \mathscr{A} \widetilde{\mathbf{Q}}^{\dagger}\right\} .
\end{aligned}
$$

And substituting (B.3) into (B.1), $\mu$ is computed as $\mu=$ $\operatorname{tr}\left(\sigma_{n_{\mathrm{d}_{2}}}^{2} \mathbf{W}_{\mathrm{D}} \mathbf{W}_{\mathrm{D}}\right)^{\dagger} / P_{\mathrm{R}}$ and using matrix inversion lemma, the solution for the optimal relay transceiver $\widehat{\mathbf{Q}}$ is obtained.

\section{Proof of Theorem 3}

By using matrix inversion lemma, the error covariance matrix in (7) becomes

$$
\begin{aligned}
\mathbf{R}_{\mathrm{E}}= & \sigma_{x}^{2} \mathbf{I}_{N_{\mathrm{s}}}-\sigma_{x}^{4} \mathscr{H}^{\dagger} \\
& \times\left[\gamma^{2} \widehat{\mathbf{G}} \mathbf{B} \mathbf{R}_{y} \mathbf{B}^{\dagger} \widehat{\mathbf{G}}^{\dagger}+\left(\sigma_{n_{\mathrm{d}_{2}}}^{2}+\sigma_{\mathrm{E}_{\mathrm{T}}}^{2} \operatorname{tr}\left(\gamma^{2} \mathbf{B} \mathbf{R}_{y} \mathbf{B}^{\dagger}\right)\right) \mathbf{I}_{N_{\mathrm{d}}}\right]^{-1} \mathscr{H} .
\end{aligned}
$$

The relay covariance matrix of signal $\mathbf{R}_{y}$ is given by

$$
\begin{aligned}
\mathbf{R}_{y} & =\mathbf{L}_{\mathrm{R}}\left[\sigma_{x}^{2} \widehat{\mathbf{H}} \mathbf{F} \mathbf{F}^{\dagger} \widehat{\mathbf{H}}^{\dagger}+\left(\sigma_{n_{\mathrm{r}}}^{2}+\sigma_{\mathrm{E}_{\mathrm{H}}}^{2} \operatorname{tr}\left(\sigma_{x}^{2} \mathbf{F} \mathbf{F}^{\dagger}\right)\right) \mathbf{I}_{N_{\mathrm{r}}}\right] \mathbf{L}_{\mathrm{R}}^{\dagger}, \\
& =\sigma_{x}^{2} \mathbf{F}^{\dagger} \mathbf{H}^{\dagger} \mathbf{H F}\left[\mathbf{F}^{\dagger} \mathbf{H}^{\dagger} \mathbf{H} \mathbf{F}+\left(\frac{\sigma_{n_{\mathrm{r}}}^{2}+\sigma_{\mathrm{E}_{\mathrm{H}}}^{2} \operatorname{tr}\left(\sigma_{x}^{2} \mathbf{F} \mathbf{F}^{\dagger}\right)}{\sigma_{x}^{2}}\right) \mathbf{I}_{N_{\mathrm{s}}}\right]^{-1} .
\end{aligned}
$$

Thus, $\mathbf{R}_{\mathrm{E}}$ is given by

$$
\mathbf{R}_{\mathrm{E}}=\sigma_{x}^{2} \mathbf{I}_{N_{\mathrm{s}}}-\sigma_{x}^{4} \mathscr{H}^{\dagger}\left[\gamma^{2} \widehat{\mathbf{G}} \mathbf{B} \mathbf{R}_{y} \mathbf{B}^{\dagger} \widehat{\mathbf{G}}^{\dagger}+\beta \mathbf{I}_{N_{\mathrm{d}}}\right]^{-1} \mathscr{H},
$$

where $\beta=\sigma_{n_{\mathrm{d}_{2}}}^{2}+\sigma_{\mathrm{E}_{\mathrm{T}}}^{2} \operatorname{tr}\left(\gamma^{2} \mathbf{B R}_{y} \mathbf{B}^{\dagger}\right)$. Applying matrix inversion lemma to (C.3) and other mathematical manipulations, we get

$$
\begin{aligned}
\mathbf{R}_{\mathrm{E}}= & \sigma_{x}^{2} \mathbf{I}_{N_{s}}-\frac{\sigma_{x}^{2} \gamma}{\beta} \mathscr{H}^{\dagger} \widehat{\mathbf{G}} \mathbf{B}\left(\frac{\gamma^{2}}{\beta} \mathbf{B}^{\dagger} \widehat{\mathbf{G}}^{\dagger} \widehat{\mathbf{G}} \mathbf{B}+\mathbf{R}_{y}^{-1}\right)^{-1} \\
& \times \sigma_{x}^{2} \mathbf{R}_{y}^{-1} \mathbf{L}_{\mathrm{R}} \widehat{\mathbf{H}} \mathbf{F} .
\end{aligned}
$$

Defining $\mathscr{J}$ as $\mathscr{J} \triangleq \sigma_{x}^{2} \mathbf{R}_{y}^{-1} \mathbf{L}_{\mathbf{R}} \widehat{\mathbf{H}} \mathbf{F} \equiv \mathbf{I}_{N_{\mathrm{s}}}$ yields

$$
\begin{aligned}
\mathbf{R}_{\mathrm{E}}= & \sigma_{x}^{2} \mathbf{I}_{N_{\mathrm{s}}}-\sigma_{x}^{2} \mathbf{F}^{\dagger} \widehat{\mathbf{H}}^{\dagger} \mathbf{L}_{\mathrm{R}}^{\dagger}\left(\frac{\gamma^{2}}{\beta} \mathbf{B}^{\dagger} \widehat{\mathbf{G}}^{\dagger} \widehat{\mathbf{G}} \mathbf{B}+\mathbf{R}_{y}^{-1}-\mathbf{R}_{y}^{-1}\right) \\
& \times\left(\frac{\gamma^{2}}{\beta} \mathbf{B}^{\dagger} \widehat{\mathbf{G}}^{\dagger} \widehat{\mathbf{G}} \mathbf{B}+\mathbf{R}_{y}^{-1}\right)^{-1} \mathscr{J}, \\
= & \left(\sigma_{n_{\mathrm{r}}}^{2}+\sigma_{\mathrm{E}_{\mathrm{H}}}^{2} P_{\mathrm{T}}\right)\left(\mathbf{F}^{\dagger} \widehat{\mathbf{H}}^{\dagger} \widehat{\mathbf{H}} \mathbf{F}+\frac{\sigma_{n_{\mathrm{r}}}^{2}+\sigma_{\mathrm{E}_{\mathrm{H}}}^{2} P_{\mathrm{T}}}{\sigma_{x}^{2}} \mathbf{I}_{N_{\mathrm{s}}}\right)^{-1} \\
& +\beta\left(\gamma^{2} \mathbf{B}^{\dagger} \widehat{\mathbf{G}}^{\dagger} \widehat{\mathbf{G}} \mathbf{B}+\beta \mathbf{R}_{y}^{-1}\right)^{-1},
\end{aligned}
$$

\section{Proof of Theorem 5}

The cost function $\mathscr{C}$ is formulated using the Lagrangian multiplier $\mu$ as

$$
\mathscr{C}=\varepsilon\left\{\mathbf{e e}^{\dagger}\right\}+\mu\left\{\operatorname{tr}\left(\mathbf{Q} \mathscr{A} \mathbf{Q}^{\dagger}\right)\right\} .
$$

To obtain the optimal relay transceiver, the derivative of the cost function with respect to $\mathbf{Q}$ is set to zero as follows:

$$
\begin{aligned}
\frac{\partial \mathscr{C}}{\partial \mathbf{Q}^{*}}= & {\left[\widehat{\mathbf{G}}^{\dagger} \mathbf{W}_{2}^{\dagger} \mathbf{W}_{2} \widehat{\mathbf{G}}+\mu \mathbf{I}_{N_{\mathrm{r}}}+\operatorname{tr}\left(\sigma_{\mathrm{E}_{\mathrm{T}}}^{2} \mathbf{W}_{2} \mathbf{W}_{2}^{\dagger}\right)\right] \mathbf{Q} \mathscr{A} } \\
& +\sigma_{x}^{2} \widehat{\mathbf{G}}^{\dagger} \mathbf{W}_{2}^{\dagger} \mathbf{W}_{1} \widehat{\mathbf{T}} \widehat{\mathbf{H}}^{\dagger}-\sigma_{x}^{2} \widehat{\mathbf{G}}^{\dagger} \mathbf{W}_{2}^{\dagger} \widehat{\mathbf{H}}^{\dagger}=0 .
\end{aligned}
$$

Solving (10), the optimal $\mathbf{Q}$ can be obtained from (22) as $\widehat{\mathbf{Q}}=\mathbf{B L}_{\mathrm{R}}$

\section{E. Proof of Theorem 6}

Using matrix inversion lemma and the fact that the error covariance is defined as $\mathbf{R}_{\mathrm{E}}=\varepsilon\left\{\mathbf{x} \mathbf{x}^{\dagger}\right\}-\mathbf{W} \varepsilon\left\{\mathbf{y} \mathbf{x}^{\dagger}\right\}$ and the Wiener filter is given by $\mathbf{W}=\left(\sigma_{x}^{-2} \mathbf{I}_{N_{\mathrm{t}}}+\mathscr{H}^{\dagger} \mathbf{R}^{-1} \mathscr{H}\right)^{-1} \mathscr{H}^{\dagger} \mathbf{R}^{-1}$, $\mathbf{R}_{\mathrm{E}}$ is expressed as

$$
\begin{aligned}
\mathbf{R}_{\mathrm{E}}= & {\left[\sigma_{x}^{-2} \mathbf{I}_{N_{\mathrm{t}}}+\left(\sigma_{n_{\mathrm{d}}}^{2}+\sigma_{\mathrm{E}_{\mathrm{T}}}^{2} \operatorname{tr}\left(\sigma_{x}^{2} \mathbf{I}_{N_{\mathrm{t}}}\right)\right)^{-1} \widehat{\mathbf{T}}^{\dagger} \widehat{\mathbf{T}}\right.} \\
& \left.+\widehat{\mathbf{H}}^{\dagger} \mathbf{Q}^{\dagger} \widehat{\mathbf{G}}^{\dagger} \mathbf{R}_{2}^{-1} \widehat{\mathbf{G}} \hat{\mathbf{H}}\right]^{-1},
\end{aligned}
$$
$\begin{array}{ll}\text { where } & \mathbf{R}_{2}=\left(\sigma_{n_{\mathrm{r}}}^{2}+\sigma_{\mathrm{E}_{\mathrm{H}}}^{2} \operatorname{tr}\left(\sigma_{x}^{2} \mathbf{I}_{N_{\mathrm{t}}}\right)\right) \widehat{\mathbf{G}} \mathbf{Q Q} \mathbf{Q}^{\dagger} \widehat{\mathbf{G}}^{\dagger}+\left(\sigma_{n_{\mathrm{d}_{2}}}^{2}+\right. \\ \left.\sigma_{\mathrm{E}_{\mathrm{G}}}^{2} \operatorname{tr}\left(\mathbf{Q} \mathscr{A} \mathbf{Q}^{\dagger}\right)\right) \mathbf{I}_{N_{\mathrm{d}}} & \end{array}$

Letting $\mathbf{R}_{\mathrm{T}}^{-1} \stackrel{N_{\mathrm{d}} \cdot}{=} \sigma_{x}^{-2} \mathbf{I}_{N_{\mathrm{t}}}+\left(\sigma_{n_{\mathrm{d}}}^{2}+\sigma_{\mathrm{E}_{\mathrm{T}}}^{2} \operatorname{tr}\left(\sigma_{x}^{2} \mathbf{I}_{N_{\mathrm{t}}}\right)\right)^{-1} \widehat{\mathbf{T}}^{\dagger} \widehat{\mathbf{T}}$ and $\mathscr{H}=\widehat{\mathbf{G}}_{\mathbf{B}} \mathbf{L}_{\mathrm{R}} \mathbf{H}$, we apply the matrix inversion lemma and get

$$
\begin{aligned}
\mathbf{R}_{\mathrm{E}} & =\mathbf{R}_{\mathrm{T}}-\mathbf{R}_{\mathrm{T}} \widehat{\mathbf{H}}^{\dagger} \mathbf{Q}^{\dagger} \widehat{\mathbf{G}}^{\dagger}\left(\mathbf{R}_{2}+\widehat{\mathbf{G}} \mathbf{Q} \widehat{\mathbf{H}} \mathbf{F} \mathbf{R}_{\mathrm{T}} \widehat{\mathbf{H}}^{\dagger} \mathbf{Q}^{\dagger} \widehat{\mathbf{G}}^{\dagger}\right)^{-1} \mathbf{G} \mathbf{Q H} \mathbf{R}_{\mathrm{T}}, \\
& =\mathbf{R}_{\mathrm{T}}-\mathbf{R}_{\mathrm{T}} \mathscr{H}^{\dagger}\left(\widehat{\mathbf{G}} \mathbf{B} \boldsymbol{\Omega} \mathbf{B}^{\dagger} \mathbf{G}^{\dagger}+\mathbf{S}\right)^{-1} \mathscr{H} \mathbf{R}_{\mathrm{T}},
\end{aligned}
$$

where $\mathbf{S}=\left[\sigma_{n_{\mathrm{d}_{2}}}^{2}+\sigma_{\mathrm{E}_{\mathrm{G}}}^{2} \operatorname{tr}\left(\mathbf{B R}_{\mathrm{y}} \mathbf{B}^{\dagger}\right)\right] \mathbf{I}_{N_{\mathrm{d}}}$, and the positive definite matrix $\boldsymbol{\Omega}$ is defined as

$$
\begin{aligned}
\Omega & \triangleq \mathbf{L}_{\mathrm{R}}\left(\sigma_{n_{\mathrm{r}}}^{2} \mathbf{I}_{N_{\mathrm{r}}}+\sigma_{\mathrm{E}_{\mathrm{H}}}^{2} \operatorname{tr}\left(\sigma_{x}^{2} \mathbf{I}_{N_{\mathrm{t}}}\right) \mathbf{I}_{N_{\mathrm{r}}}+\widehat{\mathbf{H}} \mathbf{R}_{\mathrm{T}} \widehat{\mathbf{H}}^{\dagger}\right) \mathbf{L}_{\mathrm{R}}^{\dagger} . \\
& =\mathbf{R}_{\mathrm{T}} \mathbf{F}^{\dagger} \widehat{\mathbf{H}}^{\dagger} \widehat{\mathbf{H}} \mathbf{F}\left(\mathbf{F}^{\dagger} \widehat{\mathbf{H}}^{\dagger} \widehat{\mathbf{H}} \mathbf{F}+\mathbf{R}_{\mathrm{T}}^{-1}+\sigma_{\mathrm{E}_{\mathrm{H}}}^{2} \operatorname{tr}\left(\sigma_{x}^{2} \mathbf{I}_{N_{\mathrm{t}}}\right) \mathbf{R}_{\mathrm{T}}^{-1}\right)^{-1} .
\end{aligned}
$$

Applying matrix inversion lemma again to (E.2) and other mathematical manipulations, (23) is obtained.

\section{Conflicts of Interest}

The authors declare that there are no conflicts of interest regarding the publication of this paper.

\section{Acknowledgments}

This work was supported in part by the National Research Foundation of Korea through the Korean Government (MSIP) (NRF-2016R1C1B2011921) and in part by the 
Institute for Information and Communications Technology Promotion through the Korean Government (MSIP) (no. 2016-0-00500, Cross layer design of cryptography and physical layer security for IoT networks).

\section{References}

[1] X. Tang and Y. Hua, "Optimal design of non-regenerative MIMO wireless relays," IEEE Transactions on Wireless Communications, vol. 6, no. 4, pp. 1398-1407, 2007.

[2] C. Song, K.-J. Lee, and I. Lee, "MMSE based transceiver designs in closed-loop non-regenerative MIMO relaying systems," IEEE Transactions on Wireless Communications, vol. 9, no. 7, pp. 2310-2319, 2010.

[3] C. Song, K.-J. Lee, and I. Lee, "Designs of MIMO amplify-andforward wireless relaying networks: practical challenges and solutions based on MSE decomposition," IEEE Access, vol. 5, pp. 9223-9234, 2017.

[4] H. Shen, W. Xu, and C. Zhao, "Transceiver optimization for full-duplex massive MIMO of relaying with direct link," IEEE Access, vol. 4, pp. 8857-8864, 2016.

[5] Y. Jeon, C. Song, S. Maeng, M. Park, and I. Lee, "MMSE based two-stage beamforming for large-scale multi-user MISO systems," in Proceedings of the 2016 IEEE 27th Annual International Symposium on Personal, Indoor and Mobile Radio Communications, pp. 1-6, Valencia, Spain, September 2016.

[6] P. Ubaidulla and A. Chockalingam, "Relay precoder optimization in MIMO-relay networks with imperfect CSI," IEEE Transactions on Signal Processing, vol. 59, no. 11, pp. 54735484, 2011.

[7] Z. He, W. Jiang, and Y. Rong, "Robust design for amplify-andforward MIMO relay systems with direct link and imperfect channel information," IEEE Transactions on Wireless Communications, vol. 14, no. 1, pp. 353-363, 2015.

[8] R. Mo and Y. H. Chew, "Precoder design for non-regenerative MIMO relay systems," IEEE Transactions on Wireless Communications, vol. 8, no. 8, pp. 5041-5049, 2009.

[9] F. S. Tseng, W. R. Wu, and J. Y. Wu, "Joint source/relay precoder design in nonregenerative cooperative systems using an MMSE criterion," IEEE Transactions on Wireless Communications, vol. 8, no. 10, pp. 4928-4933, 2009.

[10] C. Li, X. Wang, L. Yang, and W. P. Zhu, "A joint source and relay power allocation scheme for a class of MIMO relay systems," IEEE Transactions on Signal Processing, vol. 57, no. 12, pp. 4852-4860, 2009.

[11] M. Ding, S. Liu, H. Luo, and X. Wang, "Antenna selection for AF MIMO relay networks and the capacity scaling tendency," in Proceedings of the IEEE International Conference on Communications, pp. 1-5, Cape Town, South Africa, May 2010.

[12] Z. Fang, Y. Hua, and J. C. Koshy, "Joint source and relay optimization for a non-regenerative MIMO relay," in Proceedings of the Fourth IEEE Workshop Sensor Array and Multichannel Processing, pp. 239-243, Waltham, MA, USA, July 2006.

[13] K.-J. Lee, J. S. Kim, G. Caire, and I. Lee, "Asymptotic ergodic capacity analysis for MIMO amplify-and-forward relay networks," IEEE Transactions on Wireless Communications, vol. 9, no. 9, pp. 2712-2717, 2010.

[14] K.-J. Lee, H. Sung, E. Park, and I. Lee, "Joint optimization for one and two-way MIMO AF multiple-relay systems," IEEE Transactions on Wireless Communications, vol. 9, no. 12, pp. 3671-3681, 2010.
[15] W. Guan and H. Luo, "Joint MMSE transceiver design in nonregenerative MIMO relay systems," IEEE Communications Letters, vol. 12, no. 7, pp. 517-519, 2008.

[16] M. Ding and S. Blostein, "MIMO LMMSE transceiver design with imperfect CSI at both ends," in Proceedings of the IEEE International Conference on Communications, pp. 4369-4374, Glasgow, UK, June 2007.

[17] H.-B. Kong, C. Song, H. Park, and I. Lee, "Peak power constrained closed-form transceiver designs for MIMO AF relaying systems with direct link," in Proceedings of the IEEE Global Communications Conference, pp. 4318-4323, Austin, TX, USA, December 2014.

[18] C. T. Lin, F. S. Tseng, and W. R. Wu, "MMSE transceiver design for full-duplex MIMO relay systems," IEEE Transactions on Vehicular Technology, vol. 66, no. 8, pp. 6849-6861, 2017.

[19] N. I. Miridakis and T. A. Tsiftsis, "On the joint impact of hardware impairments and imperfect CSI on successive decoding," IEEE Transactions on Vehicular Technology, vol. 66, no. 6, pp. 4810-4822, 2017.

[20] C. Song and C. Ling, "On the diversity of linear transceivers in MIMO AF relaying systems," IEEE Transactions on Information Theory, vol. 62, no. 1, pp. 272-289, 2016.

[21] M. R. A. Khandaker and Y. Rong, "Transceiver optimization for multi-hop MIMO relay multicasting from multiple sources," IEEE Transactions on Wireless Communications, vol. 13, no. 9, pp. 5162-5172, 2014.

[22] T. Yoo and A. Goldsmith, "Capacity and power allocation for fading MIMO channels with channel estimation error," IEEE Transactions on Information Theory, vol. 52, no. 5, pp. 2203-2214, 2006.

[23] A. Dabbagh and D. Love, "Multiple antenna MMSE based downlink precoding with quantized feedback or channel mismatch," IEEE Transactions on Communications, vol. 56, no. 11, pp. 1859-1868, 2008.

[24] E. Eraslan, B. Daneshrad, and C. Y. Lou, "Performance indicator for MIMO MMSE receivers in the presence of channel estimation error," IEEE Wireless Communications Letters, vol. 2, no. 2, pp. 211-214, 2013.

[25] K.-J. Lee and I. Lee, "Diversity analysis of coded SVD schemes for MIMO spatial multiplexing systems," in Proceedings of the IEEE International Conference on Communications, pp. 4703-4707, Beijing, China, 2008.

[26] K.-J. Lee and I. Lee, "Transceiver design based on blockwise uniform channel decomposition for coded MIMO systems," IEEE Transactions on Wireless Communications, vol. 8, pp. 4241-4251, 2009.

[27] Y. Rong, X. Tang, and Y. Hua, "A unified framework for optimizing linear nonregenerative multicarrier MIMO relay communication systems," IEEE Transactions on Signal Processing, vol. 57, pp. 4837-4851, 2009.

[28] D. Palomar, J. Cioffi, and M. A. Lagunas, "Joint Tx-Rx beamforming design for multicarrier MIMO channels: a unified framework for convex optimization," IEEE Transactions on Signal Processing, vol. 51, no. 9, pp. 2381-2401, 2003.

[29] J. Laneman, D. Tse, and G. W. Wornell, "Cooperative diversity in wireless networks: efficient protocols and outage behavior," IEEE Transactions on Information Theory, vol. 50, no. 12, pp. 3062-3080, 2004.

[30] Y. Rong, "MMSE-based non-regenerative multicarrier MIMO wireless relay communications with direct source-destination link," in Proceedings of the IEEE International Conference on Communications, pp. 1-5, Dresden, Germany, 2009. 
[31] G. Liu, H. Ji, F. Yu, Y. Li, and R. Wang, "MMSE-based transceiver design in multi-user MIMO relay systems with channel correlation and estimation errors," in Proceedings of the IEEE Wireless Communications and Networking Conference, pp. 1182-1187, New Orleans, LA, USA, March 2014.

[32] C. Song, K.-J. Lee, and I. Lee, "MMSE-based MIMO cooperative relaying systems: closed-form designs and outage behavior," IEEE Journal on Selected Areas in Communications, vol. 30, no. 8, pp. 1390-1401, 2012. 


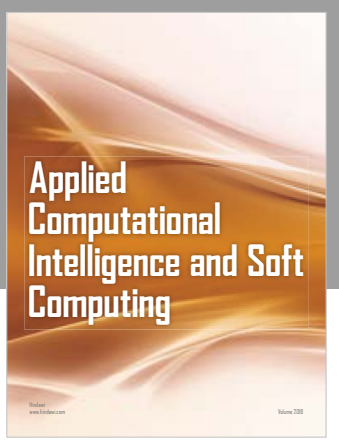

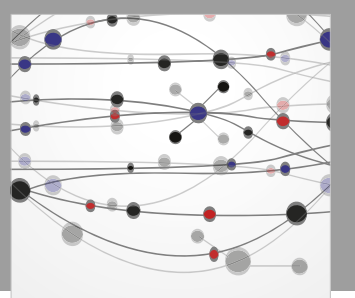

The Scientific World Journal
Submit your manuscripts at

Computing
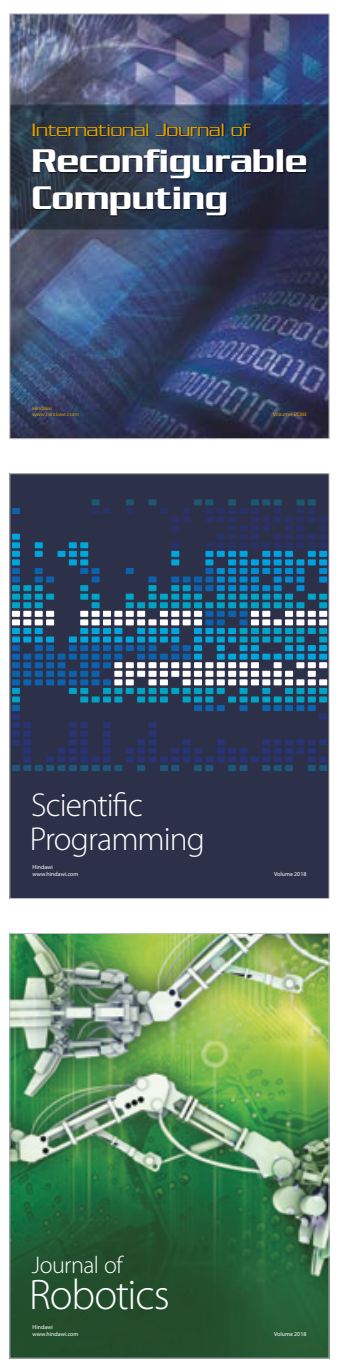

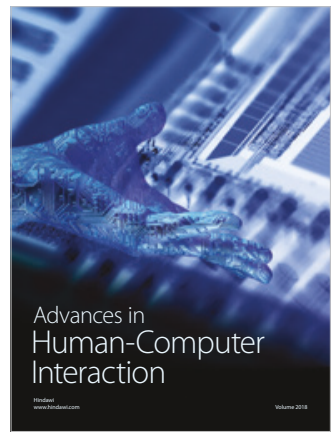

Human-Compute

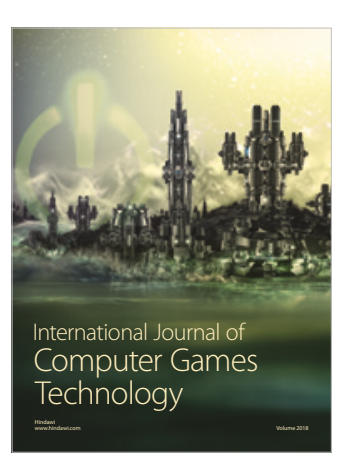

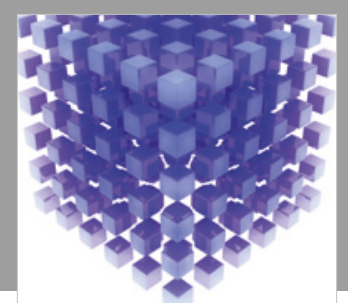

Mathematical Problems in Engineering

\section{Engincering}
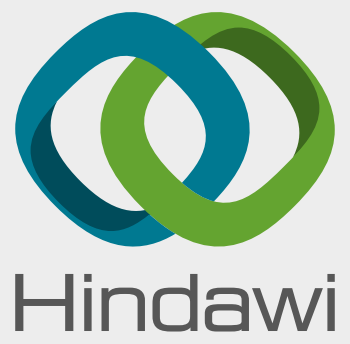

www.hindawi.com
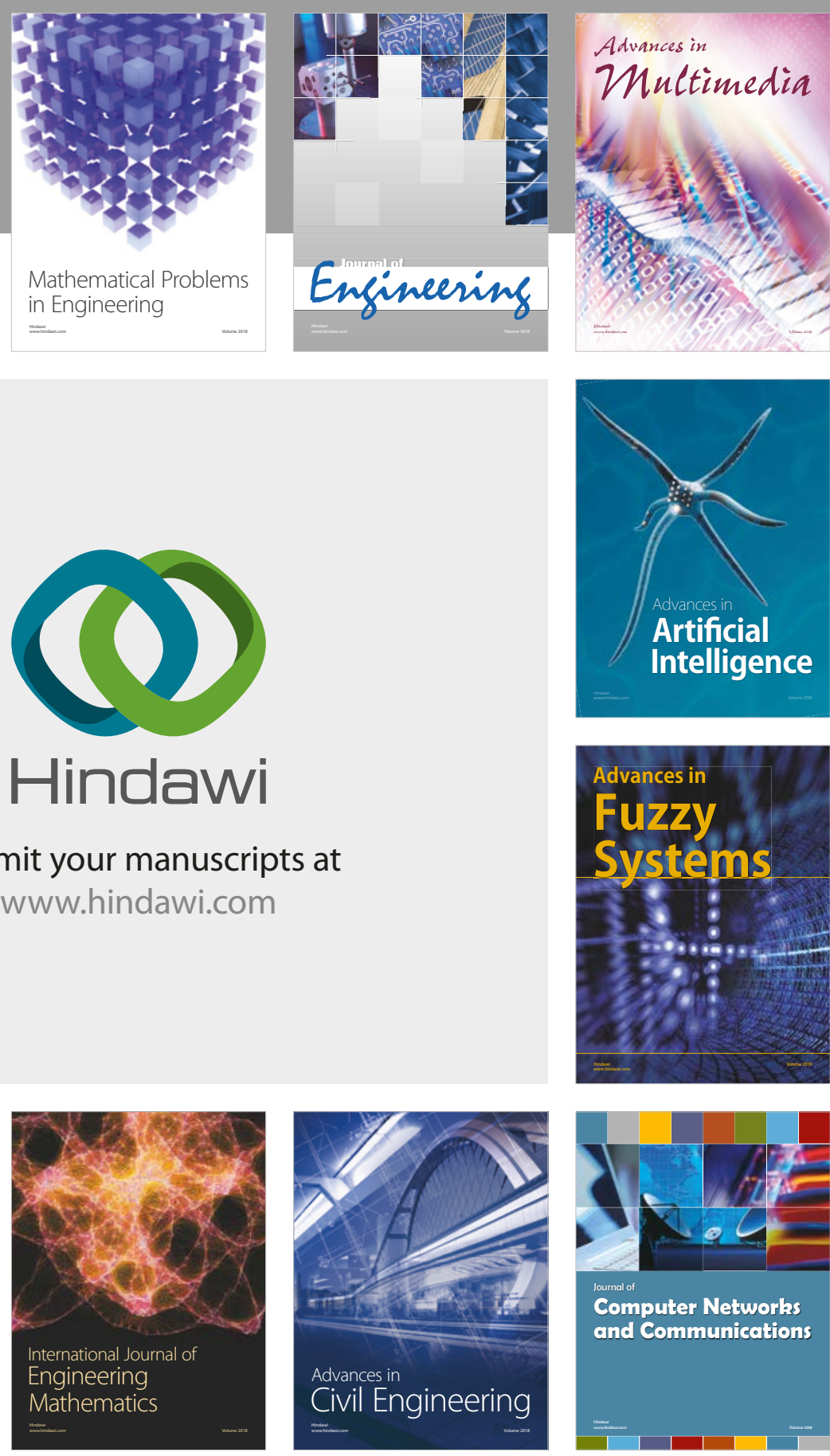

Computer Networks and Communications

Multimedia
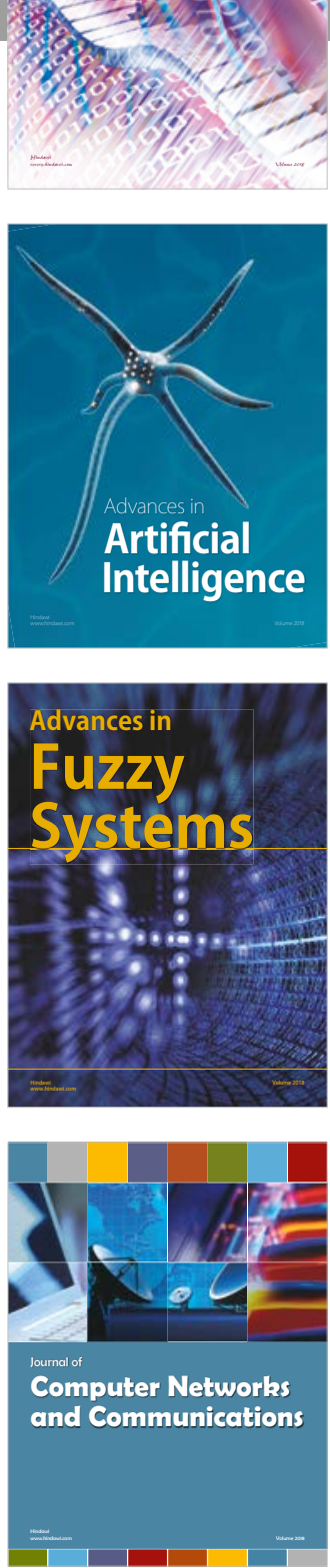

Advances in

Modelling \&

Simulation

in Engineering

interaction

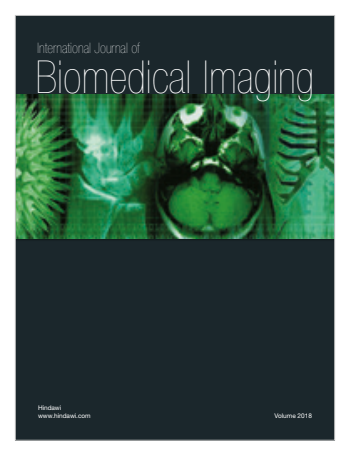

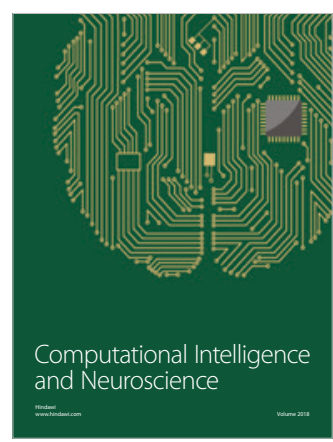

